\title{
The Spherulitic Crystallization of Isotactic Polypropylene From Solution: On the Evolution of Monoclinic Spherulites From Dendritic Chain-Folded Crystal Precursors ${ }^{1}$
}

\author{
F. Khoury
}

(October 19, 1965)

\begin{abstract}
Polypropylene can be crystallized in the form of monoclinic (Natta) spherulites from moderately concentrated solutions of the polymer in different solvents. A study is presented involving both optical and electron microscopy which has led to the characterization of the unusual structure and morphology of the dendritic precursor crystals from which such spherulites evolve, as well as the manner in which these precursors degenerate progressively into spherulites.

The overall shape of the above mentioned polypropylene dendrites approximates that of a rectangular parallelepiped (reference axes $x, y, z$, where $x / y \approx 1.1$ and $y>2 z$ ). These dendrites consist of a dense but not compact network of monolayer chain-folded lamellar branches which traverse the dendrite diagonally with respect to its rectangular $x, y$ cross section, the fold surfaces of the individual lamellar branches (i.e., those faces between which the constituent molecules in each branch fold back and forth) being normal to the $x, y$ cross section. Electron diffraction data indicate that the orientation of the $\mathbf{b}$-crystallographic axis is unique throughout the cross-hatched array of lamellar branches and is parallel to $z$, the latter axis corresponding to the direction of slowest growth of the dendrite as a whole as well as its constituent branches. It has also been deduced on the basis of the above features coupled with electron diffraction data and consideration of two different but plausible model twinned dendrites that the fold surfaces of the lamellar branches are (001) and that the angle between the ccrystallographic axis in intercrossing branches is circa $80^{\circ}$. The possible origin of this unusual mode of twinning, which involves an $80^{\circ}$ change in the orientation of the chain axes in offspring branches from that in parent branches, is briefly presented.

The process of evolution of monoclinic polypropylene spherulites from the unusually twinned dendritic crystal precursors is contrasted with the evolution of spherulites in other polymers; furthermore, the relevance of the observations presented in this study to an understanding of the origin of the previously reported atypical fine structures exhibited by monoclinic spherulites of polypropylene crystallized from the melt, is discussed.
\end{abstract}

Key Words: Chain-folded, crystal, dendritic, electron microscopy, isotactic, monoclinic, morphology, optical microscopy, polymer, polypropylene, precursor, spherulite.

\section{Introduction}

The purpose of this communication is to report on the outcome of an investigation of the nature of some crystallization habits exhibited by isotactic polypropylene when the polymer is crystallized from moderately concentrated solutions (in the range $3 / 4$ to 10 percent) in some solvents. Specifically, the relevance of various observations derived from this study to the further elucidation of the mechanism of growth and hitherto little understood origin of the atypical fine structures of spherulites of the monoclinic crystalline modification of polypropylene will be discussed in detail.

${ }^{1}$ Portions of the work described in this paper were presented before the Division of High Polymer Physics of the American Physical Society in March 1963, March 1964, and March 1965. The observations described in section 3, involving the crystallization of polypropylene from xylene and mineral oil, were made during the author's association with the Avisun Corp., Marcus Hook, Pa. All other work was carried out at NBS under partial financial support from the Advanced Research Projects Agency.
The spherulitic crystallization of polypropylene in thin films of the polymer crystallized from the melt under isothermal conditions at temperatures in the range 110 to $148{ }^{\circ} \mathrm{C}$ has been characterized by Padden and Keith $[1]^{2}$ who found that spherulites exhibiting different optical properties are formed in this temperature range. A complementary study by Keith et al., [2] of the structure of these different types of spherulites using microbeam x-ray diffraction techniques revealed that all the spherulites that are formed at temperatures above $132{ }^{\circ} \mathrm{C}$ and the majority of the spherulites formed below $132{ }^{\circ} \mathrm{C}$ possess the monoclinic crystalline structure proposed by Natta et al., [3] for isotactic polypropylene. ${ }^{3}$

\footnotetext{
${ }^{2}$ Figures in brackets indicate the literature references at the end of this paper.

${ }^{3}$ The monoclinic unit cell parameters are: $\mathbf{a}=6.65 \AA, \mathbf{b}=20.96 \AA, \mathbf{c}=6.50 \AA, \beta=99^{\circ} 20^{\prime}$. Four chains in a $3_{1}$ helical configuration pass through the unit cell which contains 12 mon omer units. The axes of the helical chains are oriented parallel to the c-axis of the cell [3].
} 
The optical characteristics (birefringence) of these monoclinic spherulites were found to depend on the temperature at which they are formed. Briefly, Padden and Keith [1] point out that the majority of the monoclinic spherulites grown below $132{ }^{\circ} \mathrm{C}$ and above $138{ }^{\circ} \mathrm{C}$ are respectively positively birefringent and negatively birefringent. While these are the predominant types of monoclinic spherulites formed in these temperature ranges, an appreciable number of "mixed" ${ }^{4}$ spherulites of the same crystalline modification are also formed in these temperature ranges. Further, most of the monoclinic spherulites formed in the range 134 to $138{ }^{\circ} \mathrm{C}$ are of the mixed type.

The exact origin of the differences in the optical properties of the various types of monoclinic spherulites is not known. The preferred radial crystallographic orientations in both the positive and negative spherulites, as deduced from microbeam x-ray diffraction data, are indistinguishable. According to Keith et al., [2] in both these types of spherulites the ccrystallographic axis, and hence the axes of the helical chains, are oriented at an angle of 65 to $70^{\circ}$ to the radius. The $\mathbf{b}$-axis orientation is tangential.

In addition to the predominantly positive and fewer mixed monoclinic spherulites which grow at temperatures below $132{ }^{\circ} \mathrm{C}$, Padden and Keith [1] observed that a few negatively birefringent spherulites are formed also in the same temperature range (110 to $132{ }^{\circ} \mathrm{C}$ ). These rarer spherulites possess an apparently pseudohexagonal metastable crystalline structure $[2,4,5]$. The axes of the chain molecules are normal to the radial direction in such spherulites $[1,2]$.

Spherulites of the more common monoclinic crystalline modification and the rarer hexagonal crystalline modification of polypropylene exhibit highly contrasting fine structures. The morphology of the rarer hexagonal spherulites is similar to that commonly exhibited by spherulites in numerous other polymers [6]. Surface replicas of hexagonal spherulites formed in thin films of polymer reveal, as described by Geil [6], a radiating fine structure consisting of thin lamellar structural units which extend radially outwards from the central regions of the spherulites. The crystallographic orientation in these spherulites as derived from both their optical properties as well as the microbeam x-ray diffraction experiments referred to above (which indicate that the chain molecules are oriented normal to the radius), coupled with the uniformity in thickness of the radiating lamellae normal to the radius, corroborate that these lamellae are most probably chain-folded single crystal structural units. The fold surfaces (i.e., the surfaces between which the molecules are folded regularly back and forth) of the lamellae are parallel to the radial direction as has been established in spherulites of other polymers, e.g., polyethylene, polyoxymethylene, and poly(4-methylpentene-1) [7].

\footnotetext{
${ }^{4}$ These spherulites do not exhibit symmetrical extinction characteristics between crossed polarizers; individual spherulites exhibit, randomly, positive birefringence, negative birefringence, or irregular transitions from one sign of birefringence to another along different radial paths.
}

In contrast with the radiating chain-folded lamellar morphology which, as indicated above, is characteristic of spherulites of numerous polymers, as well as those of the pseudohexagonal crystalline modification of polypropylene, spherulites of the monoclinic crystalline modification of polypropylene exhibit highly complex morphologies the nature and origin of which have hitherto been little understood. Brief descriptions of the morphology of such spherulites have been presented by Bailey [8] (see also Keller [9]) and Geil [10]. Bailey [8] has described spherulites which exhibit a cross-hatched morphology consisting apparently of interwoven or intercrossing radially and tangentially oriented "fibrils." Geil [10] describes positive and negative monoclinic spherulites as consisting of radially oriented ribbonlike or ropelike structural units which exhibit a transverse ribbed or corrugated 'morphology; apparently no specific morphological differences between the positively and negatively birefringent types of monoclinic spherulites have been observed.

Additional evidence of the unusual morphologies associated with the crystallization of polypropylene in its monoclinic crystalline modification has been further reported by Geil [11]. This evidence has been derived from a study of the morphology of extremely thin films of polypropylene crystallized by slow cooling from the melt on a substrate. Examination under the electron microscope revealed that those portions of the film which crystallized in the pseudohexagonal crystal modification consisted of thin ( 150 A thick) lamellar chain-folded crystal layers iying parallel to the substrate. Frequent instances of spiral development of superposed layers initiated by screw dislocations in basal lamellae were observed. In contrast, those regions of the polymer film which crystallized in the monoclinic modification were found to consist of "incipient spherulites" various portions of which exhibited differing morphologies. In some regions of these structures a distinctly cross-hatched fine structure akin to that reported by Bailey [8] was observed. Geil points out that the narrow structural units which may be seen to intercross at right angles within limited areas appear to be thicker normal to the substrate on which they grew than they are wide. The possibility is indicated by Geil [11] that they may be chain-folded single crystals the fold surfaces of which are oriented normal to the plane of the film. In other regions of these "incipient spherulites" individual broad ribbonlike structures were discerned which exhibited in their thicker parts a transverse striated fine structure, reminiscent of that exhibited by the constituent radiating units in positive and negative monoclinic spherulites [10].

The unorthodox morphologies of isotactic polypropylene in which the polymer crystallizes in its more common monoclinic crystalline modification clearly suggested that some fundamental differences must exist between the mechanism of evolution of monoclinic spherulites of that polymer and the mechanism leading to the formation of polymer spherulites that exhibit the more conventional radiating lamellar 
fine structures. The original objectives of the present study were to (a) investigate the nature of the habits and fine structures of crystalline aggregates that hot moderately concentrated solutions of polypropylene yield in suspension in solvent when they are cooled to lower temperatures, and (b) determine whether any information relevant to the elucidation of the origin of the complex morphologies exhibited by the relatively intractable melt-grown monoclinic spherulites could be derived from the structural characterization of crystalline aggregates grown from solution (under different conditions) that are more accessible to detailed morphological investigations.

It will first be shown in what ensues that polypropylene can be readily crystallized from solution in the form of monoclinic spherulites in suspension. Experiments will subsequently be described, the out come of which has been to provide a detailed insight into the unorthodox nature of the crystal precursors from which monoclinic spherulites evolve when formed from solution. The direct relevance of the observations derived from the latter experiments to an understanding of the origin of the atypical fine structures of melt-grown monoclinic spherulites will be pointed out. Finally, the nature of an underlying fundamental phenomenological difference between the mechanism of formation of spherulites of the monoclinic crystalline modification of polypropylene and that of polymer spherulites which exhibit the more typical radiating lamellar or fibrillar morphologies will be discussed.

\section{Experimental Procedures}

\subsection{Materials}

Isotactic polypropylene in powder form having a number average (osmotic) molecular weight of 98000 [12] was used in all the experiments. The solvents used were: mineral oil (medicinal grade, specific gravity according to manufacturer $0.880-0.900$ at $15.6{ }^{\circ} \mathrm{C}$ ), xylene (A.C.S. reagent grade, boiling range 139 to $139.3{ }^{\circ} \mathrm{C}$ ) and amyl acetate (purified). The xylene and amyl acetate were distilled before use.

\subsection{Solutions}

Unless otherwise stated, solution concentrations are expressed throughout as weight (grams) of polymer per volume $\left(100 \mathrm{~cm}^{3}\right)$ of solvent at room temperature. In general polymer solutions were prepared by adding the polypropylene powder to solvent at room temperature in a Pyrex flask and then immersing the flask and its contents for half an hour in a bath held isothermally at an appropriate elevated temperature, i.e., at $180^{\circ} \mathrm{C}$ for the mineral oil preparations, and at $140{ }^{\circ} \mathrm{C}$ for both the xylene and amyl acetate preparations. The resulting solutions were then either cooled continuously to room temperature (sec. 3.1) or transferred to constant temperature baths held at lower temperatures (sec. $3.2 \mathrm{a} ; 4)$. Details pertaining specifically to some individual experiments are given in appropriate parts of the paper.

\subsection{Optical Microscopy}

With the exception of some experiments described in section $3.2 \mathrm{~b}$, the various structural entities precipitated from solution under different conditions were examined under the optical microscope at room temperature while still in suspension in liquid using either polarizing or phase contrast optics. In the latter case contrast was often enhanced by either exchanging or highly diluting the original solvent in which the various structures were in suspension at room temperature with a liquid of appropriate refractive index. Cargille Refractive Index Liquids were used in such instances. Unless otherwise stated, all optical micrographs shown in the paper represent the types of structures that will be discussed as they variously appear when seen in different perspectives (we refer specifically here to structures that are not spherulites) while in suspension in a liquid medium.

\subsection{Electron Microscopy}

A JEM $6 \mathrm{~A}^{5}$ electron microscope was used in the study described in section 4 . The precautions required in examining the crystals described therein, in transmission, and the technique used in replicating their surfaces are presented in the selfsame section.

\section{Results and Discussion}

\subsection{Preliminary Evidence of the Spherulitic Crystal- lization of Polypropylene From Solution in Its Mono- clinic Crystalline Modification}

Exploratory experiments revealed that when hot 3/4-percent and 10 percent solutions of polypropylene in mineral oil or xylene are cooled continuously to room temperature at $\sim 0.5{ }^{\circ} \mathrm{C} / \mathrm{min}$, the polymer crystallizes in the form of spherulites in suspension in solvent.

The mineral oil solutions yielded only positively birefringent monoclinic spherulites, examples of which as seen under the polarizing microscope while still in suspension in solvent are shown in figures 1 and 2 .

In contrast, the xylene solutions yielded spherulites exhibiting various birefringent properties depending on the initial polymer concentration. Thus, whereas all the spherulites grown from the $3 / 4$ percent xylene solution were negatively birefringent (fig. 3), the 10 percent xylene solution yielded on cooling a preponderance of spherulites of different sizes exhibiting "mixed" birefringent characteristics as well as a few smaller distinctly negatively birefringent spherulites. The mixed spherulites were essentially of two general types, namely, some which exhibit an overall irregular change from positive birefringence in the central regions to negative birefringence nearer the periphery,

${ }^{5}$ Instrument identified by trade name in order to adequately specify equipment used Such identification in no way implies endorsement or recommendation by the National Bureau of Standards. 


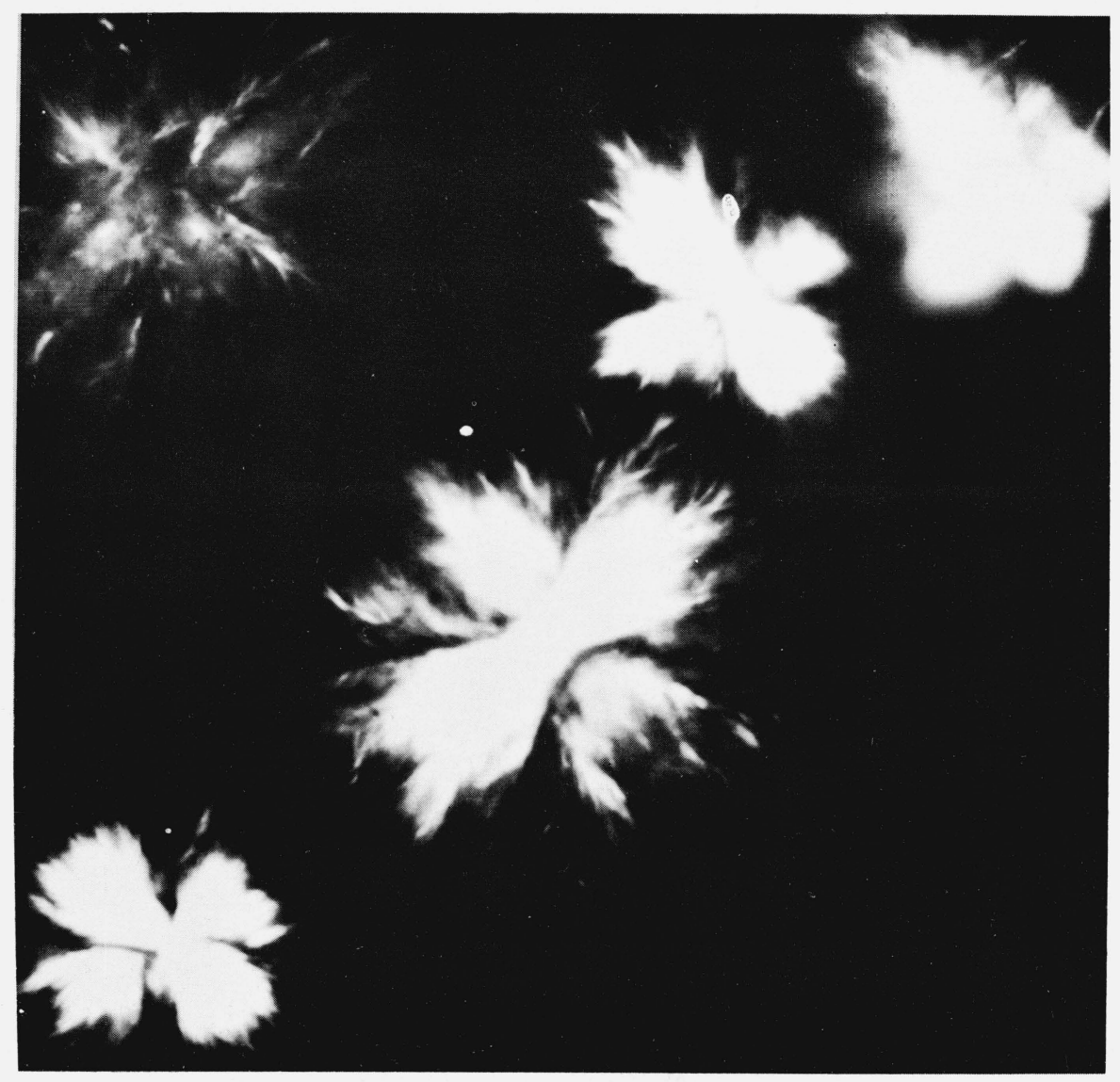

FIGURE 1. Positively birefringent spherulites grown on cooling a 0.75 percent solution of polypropylene in mineral oil.

Crossed polarizers, $\times 600$.

and others which exhibit asymmetric birefringent characteristics along different radial paths (see footnote 3). Examples of spherulites grown from a 10 percent xylene solution are shown in figure 4 .

Wide-angle x-ray diffraction patterns (powder) obtained from samples consisting of spherulites formed from the $3 / 4$ percent and 10 percent xylene solutions were characteristic of the monoclinic crystalline modification of polypropylene proposed by Natta et al., [3]. An x-ray powder pattern obtained from a sample of the negatively birefringent spherulites formed from the more dilute xylene solution is shown in figure 5 in which the four prominent 110 , 040,130 , and composite $111,13 \overline{1}, 041$ rings characteristic of monoclinic polypropylene may be readily detected.

The various solution-grown spherulites described briefly above are by no means rigid structures. They undergo varying degrees of deformation after elimination of the solvent by direct drying (xylene preparations) or by suitable extraction (mineral oil preparations). The extent of deformation to irregular shapes was pronounced in the less compact spherulites grown from the dilute polymer solutions. The spherulites grown from the 10 percent xylene solution, however, underwent only some radial shrinkage on drying while still retaining their spherical shape. Examples of spherulites removed from the latter preparation as they appear under oblique incident illumination after drying are shown in figure 6.

Further study aimed specifically at determining the origin of the different birefringent characteristics of the various types of spherulites referred to above was not attempted. It is clear, however, that the birefringent characteristics of individual spherulites grown from the 10 percent xylene solution depend on the temperature range within which they develop during the cooling cycle. The available observations indicate that lower crystallization temperatures lead to the formation of negatively birefringent spherulites, as evidenced by the fact that the smallest spherulites formed in this preparation were negatively birefringent. This is further substantiated by the, albeit irregular, change in sign of birefringence (from positive in the interior to negative nearer the periphery) exhibited by many of the larger "mixed" spherulites. That lower 


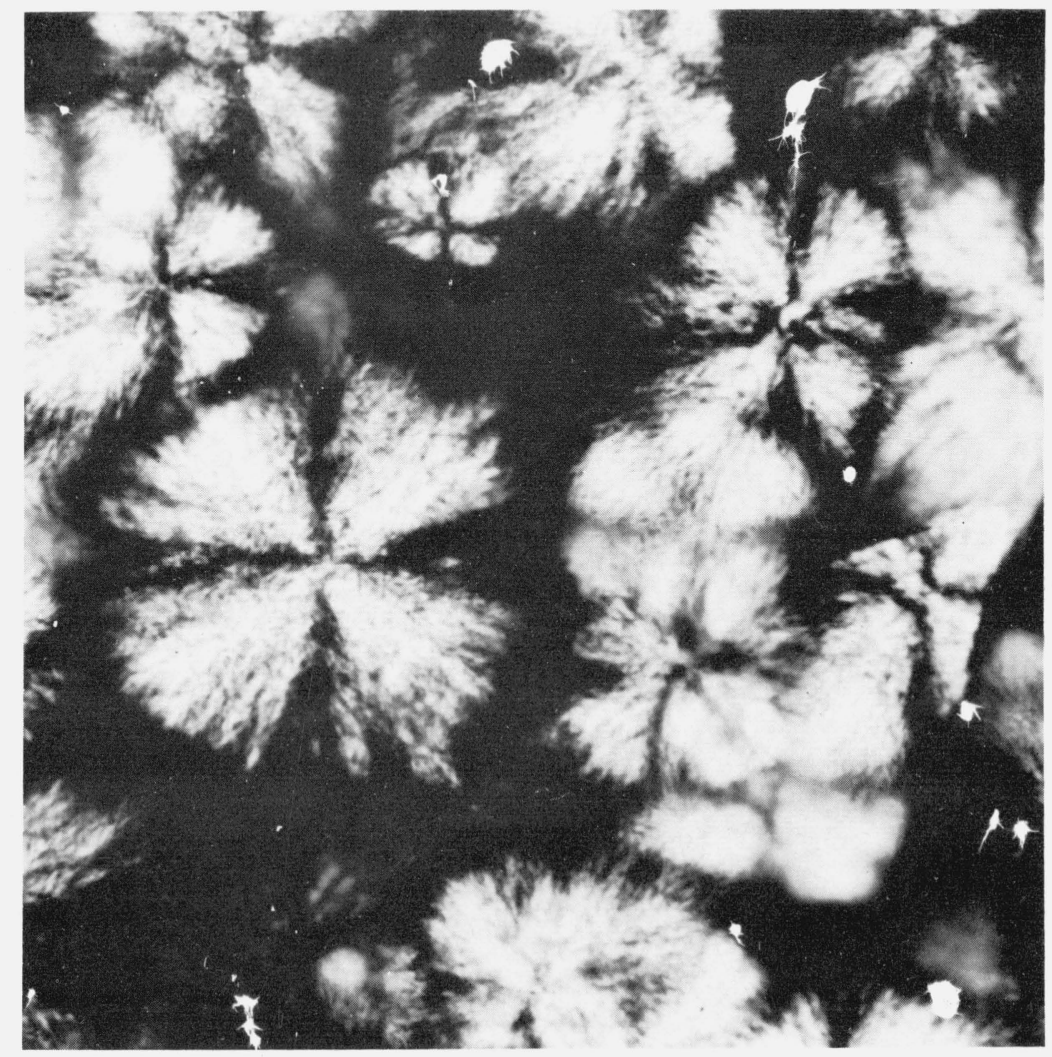

Figure 2. Positively birefringent spherulites grown on cooling a 10 percent solution of polypropylene in mineral oil. Crossed polarizers, $\times 220$.

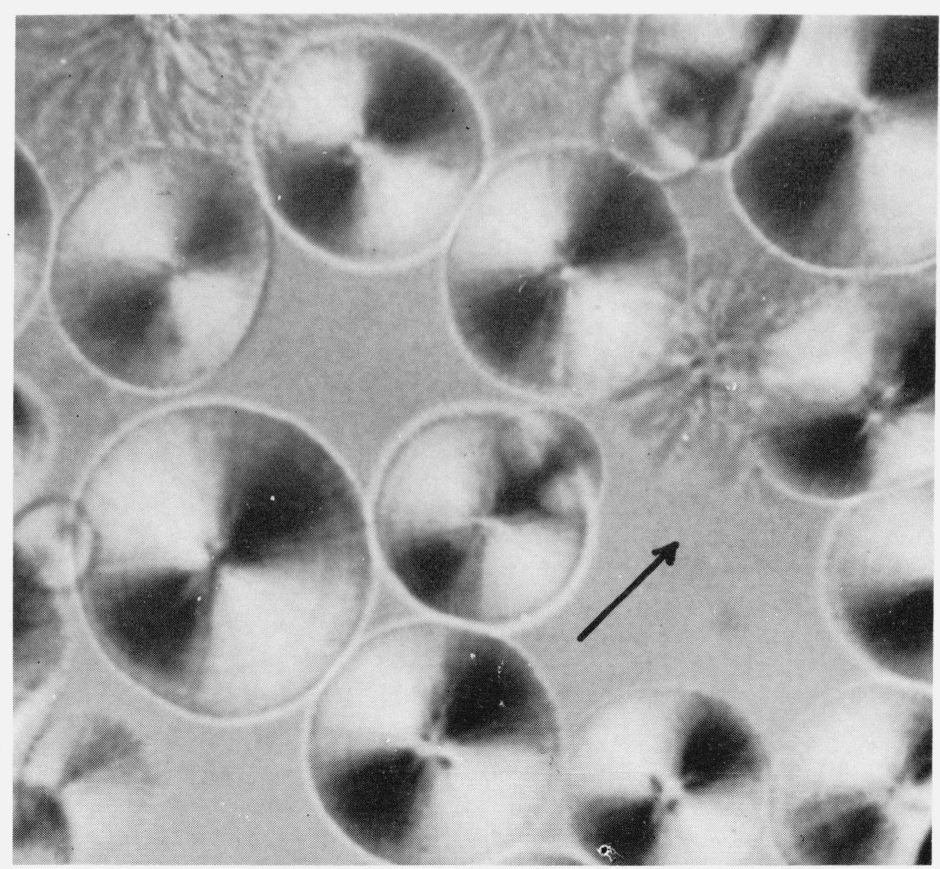

FIGURE 3. Negatively birefringent spherulites grown on cooling a 0.75 percent solution of polypropylene in xylene.

Crossed polarizers and $1 / 4 \lambda$ compensator (slow direction of vibration of compensator indicated by arrow), $\times 370$. 


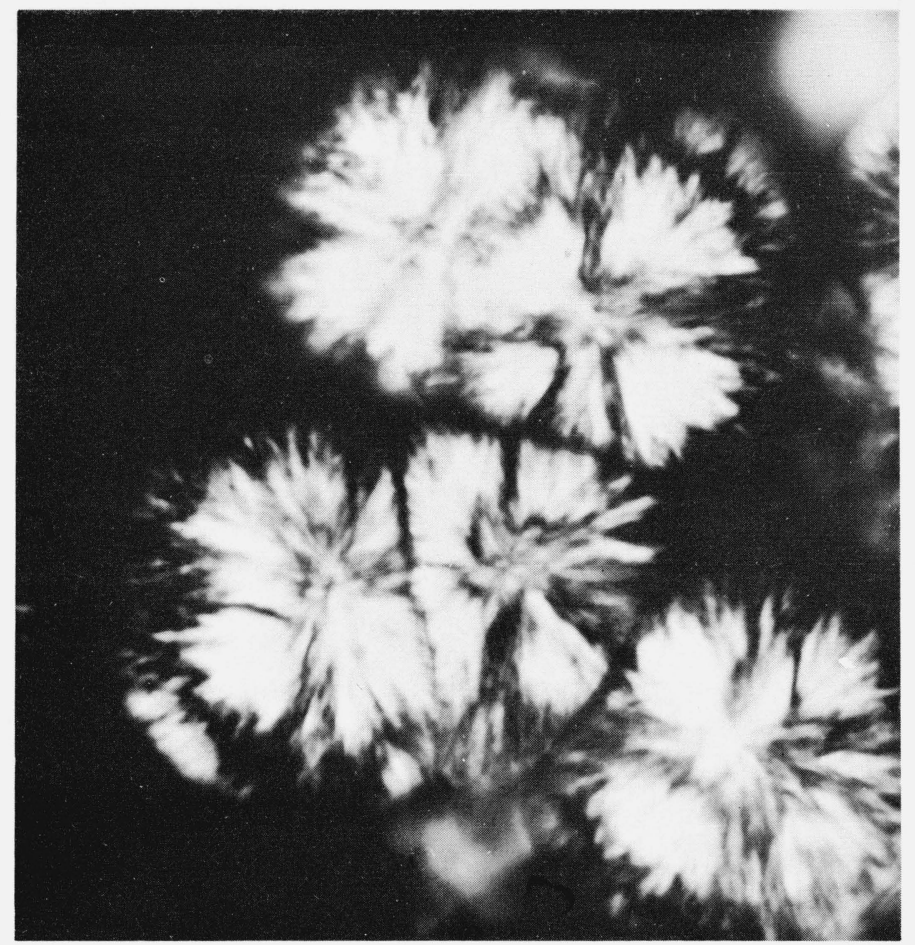

FIGURE 4. Spherulites exhibiting "mixed" birefringent characteristics grown on cooling a 10 percent solution of polypropylene in xylene. Crossed polarizers, $\times 470$.

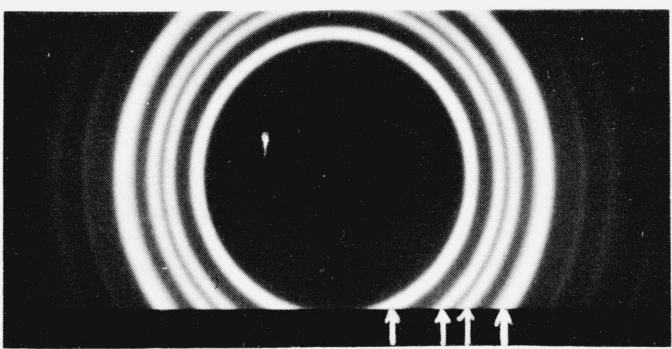

FIGURE 5. Wide angle $x$-ray powder pattern obtained from a sample of negatively birefringent spherulites formed on cooling a 0.75 percent solution of polypropylene in xylene.

Vertical bars identify inner $110,040,130$ and composite $111,13 \overline{1}, 041$ rings characteristic of the monoclinic (Natta) crystalline modification of polypropylene.

temperatures of crystallization lead to the formation of negatively birefringent monoclinic spherulites from a 10 percent xylene solution, was further substantiated by the fact that when such a solution is rapidly quenched in liquid nitrogen (rate of cooling to room temperature $\sim 44{ }^{\circ} \mathrm{C} / \mathrm{min}$ ) only negatively birefringent spherulites are formed.

\subsection{On the Nature of the Early Stages of Formation of Monoclinic Spherulites From Solution}

a. The Habits of Polypropylene Crystallized From 3/4 percent Solutions of the Polymer in Mineral Oil Under Isothermal Conditions $\left(80\right.$ to $\left.112{ }^{\circ} \mathrm{C}\right)$

At 80 and $90{ }^{\circ} \mathrm{C}$ the polymer crystallizes very rapidly. The solutions yield positively birefringent mono-

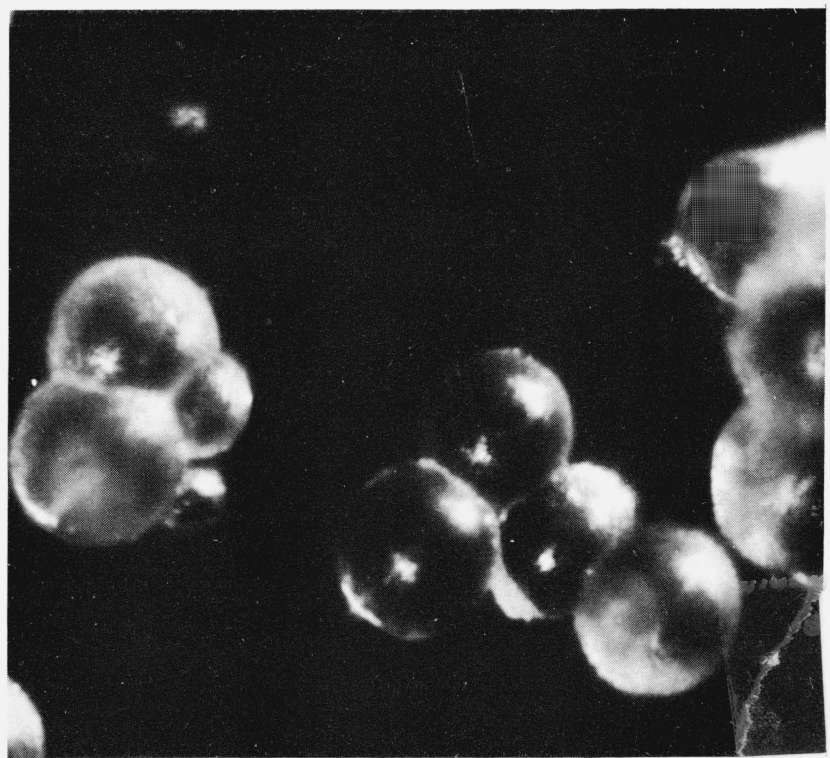

Figure 6. Spherulites grown on cooling a 10 percent solution of polypropylene in xylene.

They are depicted as seen under oblique (unpolarized) incident illumination after havin dried in air at room temperature. $\times 270$.

clinic spherulites similar to those shown in figure 1 . At higher temperatures, however, in the range 105 to $112{ }^{\circ} \mathrm{C}$ individual solutions yield in addition to relatively more compact positively birefringent monoclinic 


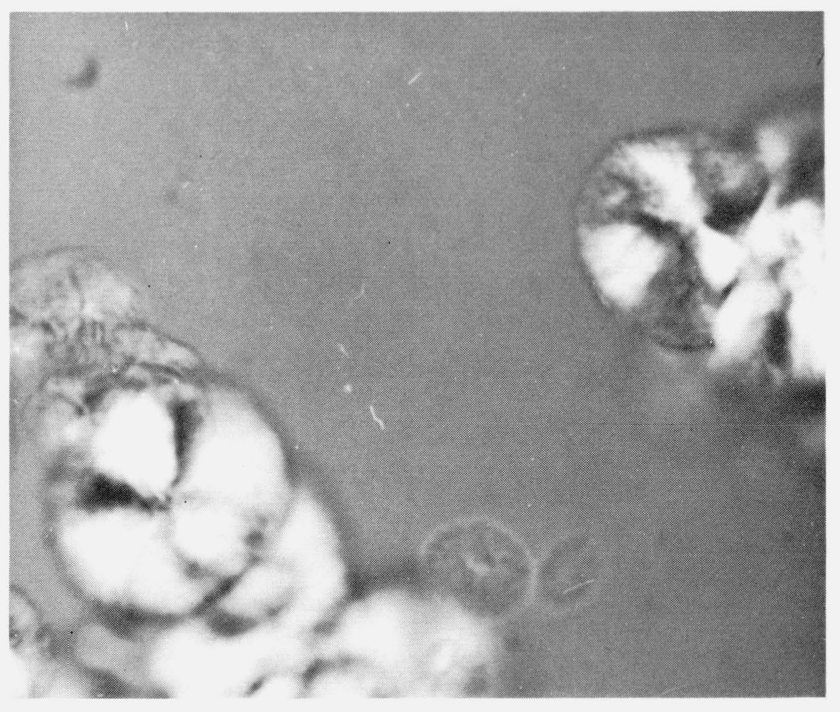

FIGURE 7. Positively birefringent spherulites and smaller aspherical aggregates grown from a 0.75 percent solution of polypropylene in mineral oil at $112^{\circ} \mathrm{C}$.

Polarizers crossed at $80^{\circ}, \times 370$

spherulites of different sizes (fig. 7), numerous smaller rigid nonspherulitic monoclinic crystalline aggregates ${ }^{6}$ which exhibit differing but related habits. The respective shapes of these various aggregates were readily deduced by observing them (after crystallization was completed and the solutions cooled to room temperature) as they tumbled, or were induced to tumble slowly, in suspension in liquid under the optical microscope in transmission either in unpolarized light or more appropriately using phase contrast optics.

An example is shown in figure 8 of an aggregate typical of many that are formed in the temperature range 105 to $112{ }^{\circ} \mathrm{C}$. These aggregates exhibit characteristically contrasting profiles when viewed along different directions. Observing such structures as they tumbled in a liquid medium revealed that they possess a plane of preferential development. When they are oriented so as to occupy a maximum surface area in the field of view of the microscope they exhibit, as may be seen in figure 8 , an essentially elliptically shaped profile. For the sake of convenience in what follows, the major and minor axes of the aggregate cross section depicted in figure 8 will be referred to below as the $(x)$ and $(y)$ axes, respectively, and the axis normal to the $(x, y)$ plane the plane of preferential development) as the $(z)$ axis. When such an aggregate is viewed along any axis lying in the $(x, y)$ plane (i.e., if the aggregate shown in figure 8 is rotated through $90^{\circ}$ from the position depicted therein about any axis normal to the microscope axis), it exhibits an essen-

\footnotetext{
${ }^{6}$ The term aggregate is used throughout this paper in a generic sense to denote any structural entity which has grown from solution and which could not be outrightly characterized as being an individual coherent crystal or a spherulite. Different types of aggre. gates will be differentiated by suitable adjuncts (e.g., elliptical, apparent or pseudopoly. hedral, etc.) referring variously to distinguishing shape characteristics.
}

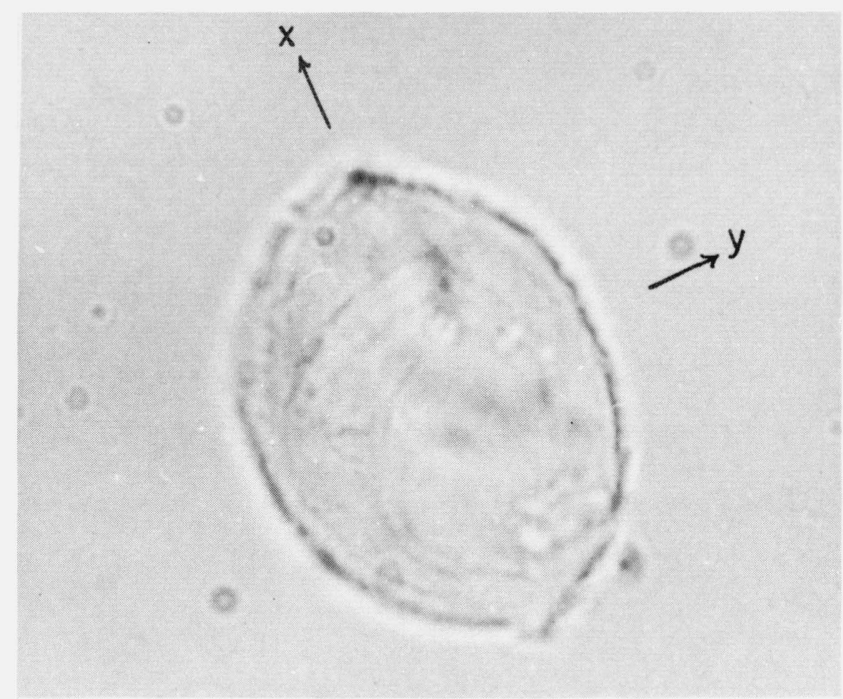

Figure 8. Crystalline aggregate grown from a 0.75 percent solution of polypropylene in mineral oil at $112^{\circ} \mathrm{C}$.

Note elliptical profile of aggregate when seen as depicted here along an axis $(z)$ at right angles to its plane $(x, y)$ of preferential development. Unpolarized light. $\times 1.930$,

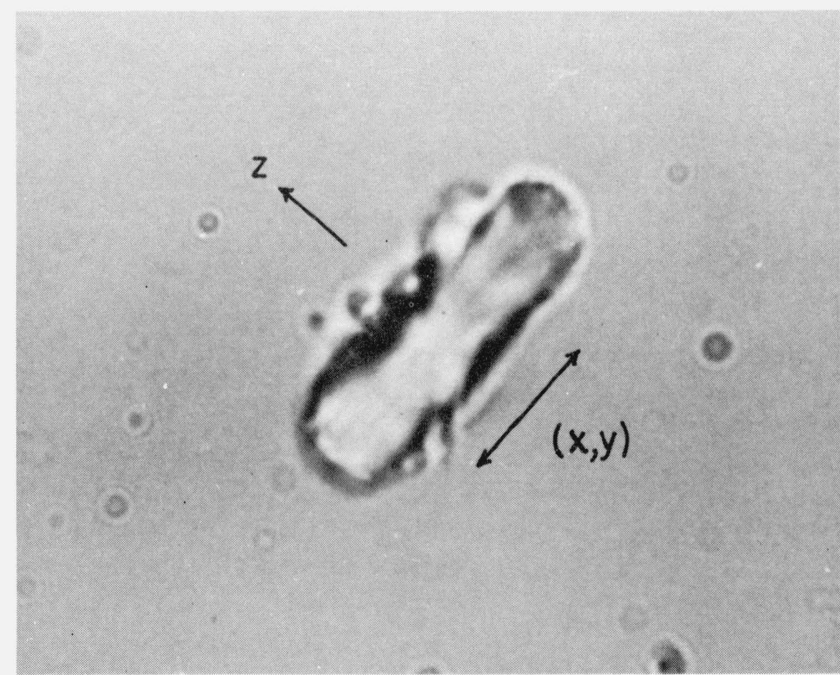

Figure 9. Aggregate similar to that shown in figure 8 but viewed edge-on along an axis parallel to its plane $(\mathrm{x}, \mathrm{y})$ of preferential development.

Unpolarized light, $\times 1.930$.

tially incipient sheaflike appearance as shown in figure 9. Overall growth along the $(z)$ direction is substantially slower than in the $(x, y)$ plane, the relative disparity in growth being roughly characterized by the following overall $(x, y, z)$ dimensions: $\sim 30 \mu \times 20 \mu$ $\times 5 \mu$ (i.e., $x: y: z \sim 6: 4: 1$ ). In actual fact these aggregates are somewhat thicker along the $(z)$-direction in the peripheral regions than at the center. This is more clearly revealed by examining these structures under the phase contrast microscope, as may be 


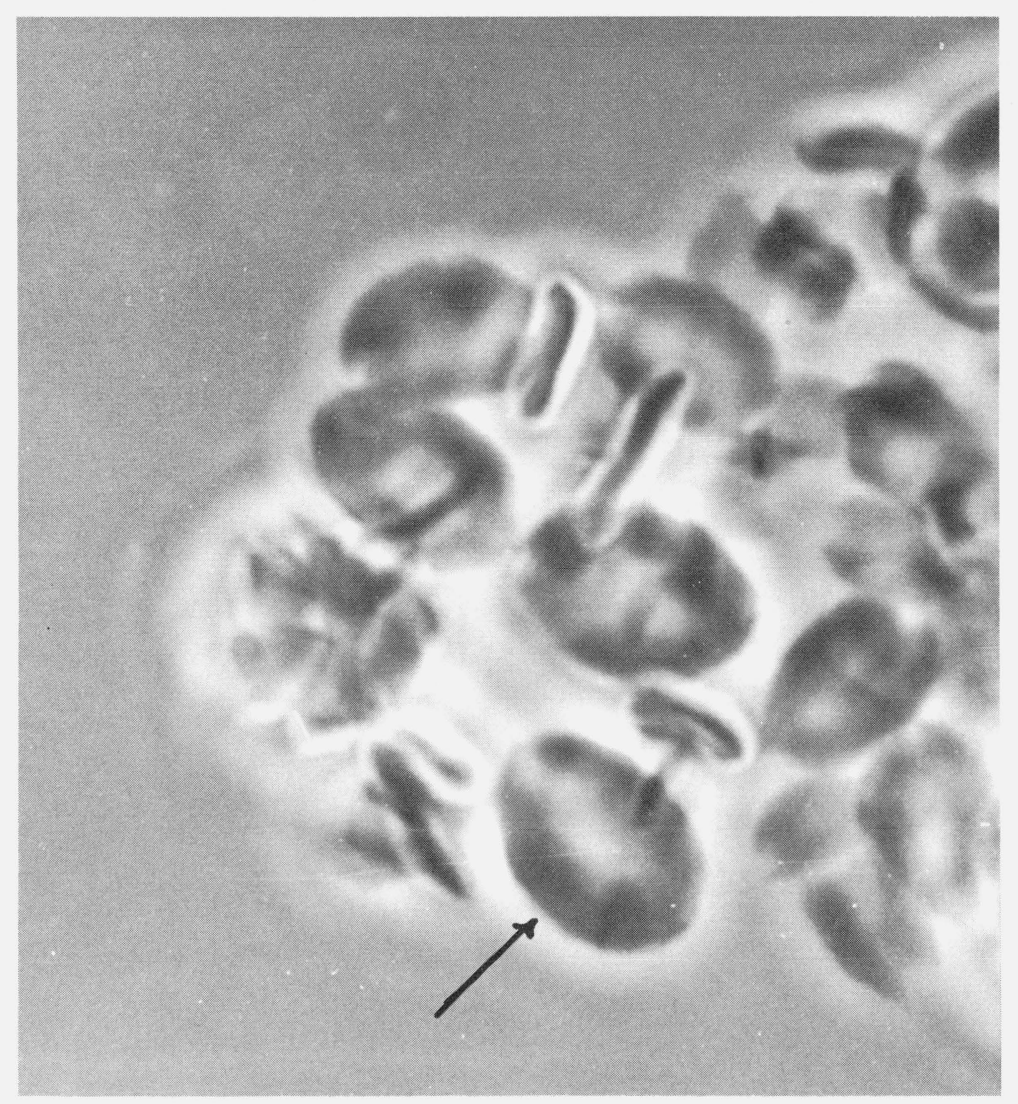

FIGURE 10. Cluster of variously oriented structures grown from a 0.75 percent polypropylene solution in mineral oil at $112^{\circ} \mathrm{C}$.

Note optically denser peripheral regions of elliptical aggregate (arrow). Phase Centrast, $\times 1450$.

seen in figure 10 , in which the distinctly darker peripheral regions in the appropriately oriented "elliptical" aggregate present in the field of view can be clearly discerned.

In addition to the type of aggregate described above, still smaller aggregates exhibiting different but related habits are also formed under the same conditions of crystallization. Examples typical of these different aggregates, as seen under the phase contrast microscope, are shown in figure 11. They are essentially planar structures which exhibit respectively the characteristic profiles seen in figure 11 (a to d) when viewed along an axis normal to their plane of preferential development.

The smallest aggregates (fig. 11a), which are relatively rare, exhibit parallel lateral edges and a distinctly concave profile at the extremities of their longer axis. This latter aspect is also evident in the slightly larger structures (fig. 11b) in which, however, the longer lateral edges are slightly convex. The lateral sides of the still larger structures (fig. 11c, d) are pronouncedly convex and these aggregates also exhibit a distinctly re-entrant or notched appearance at opposite extremities along the bisectrix parallel to their direction of preferred overall growth. As mentioned earlier, the aggregate types shown in figure 11
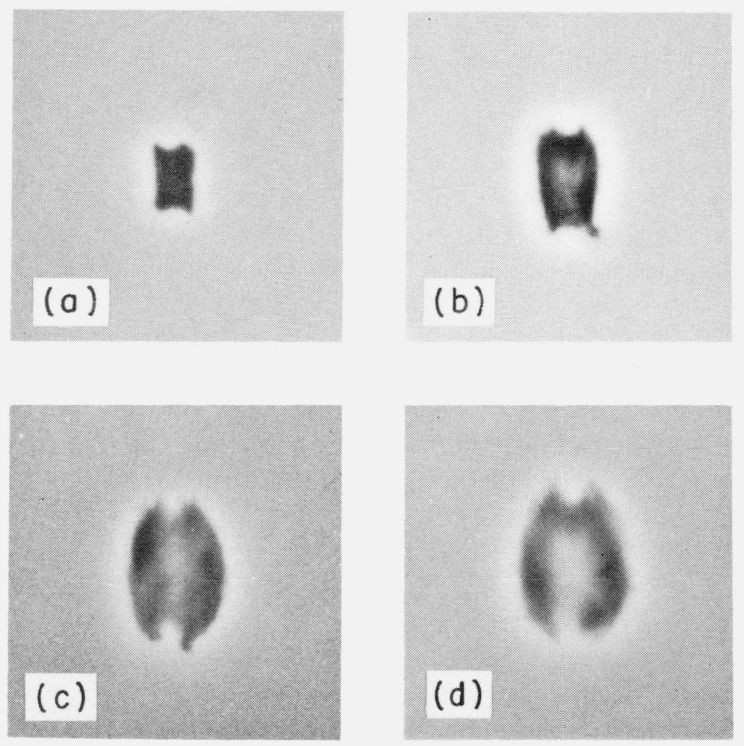

FIGURE 11. Examples of other aggregates also formed from a 0.75 polypropylene solution in mineral oil at $112^{\circ} \mathrm{C}$.

Each is depicted as seen along an axis at right angles to its plane of preferential development. Note pronounced concavity of shorter edges of aggregates in (a), (b), as well as the pronounced convexity of the lateral edges and notched appearance at opposite extremities of aggregates in (c), (d). Phase Contrast. $\times 1.050$. 
are thinner $(<5 \mu)$ than they are long or broad. They, too, however, as in the case of the elliptical structures described above, are slightly thicker in the peripheral regions than at the center. They all exhibit incipient sheaflike appearances when viewed on edge (i.e., along any axis in the plane of the respective cross sections depicted in figure 11). A view is shown in figure 12 including a few "notched" aggregates of the type shown in figure 11c, d lying flat on a substrate and several other nonspherulitic aggregates similar to those described above, oriented with their plane of preferential growth parallel to the axis of the phase contrast microscope.

A comparison of the relative sizes and corresponding habit features of the types of aggregates shown in figure 11 which had all formed under the same conditions as the elliptical structures shown in figures 8 and 10, indicates an evident size-habit trend (increased convexity of the lateral sides and progressive filling in of the re-entrant extremities) which strongly suggested that these structures might well represent early successive stages in the formation of the larger "elliptical" structures (figs. 8 to 10), but further development of which had been arrested due to depletion of the polymer from the solution. By the same token, since the spherulites (fig. 7) that are formed under the same conditions as the types of aggregates described above were larger than the elliptical structures formed at the same temperatures in individual solu- tions, the possibility was envisaged that the elliptical and other aggregates are representative of the early successive transient structures that develop progressively during the initial stages of growth of individual monoclinic spherulites. This was confirmed by some experiments described below.

\section{b. Direct Microscopic Observations of the Growth of Monoclinic Spherulites From Concentrated Solutions of Polypropylene in Mineral Oil}

The procedure adopted in these experiments was facilitated by the low volatility of the mineral oil at the temperatures at which the experiments were carried out. Appropriate weights of powdered polypropylene and mineral oil were deposited on a microscope slide at room temperature. The polymersolvent mixture was then covered with a thin coverglass and placed in the heating chamber of a Leitz heating stage (Model 350) mounted on the rotating stage of a phase contrast microscope. The temperature of the heating stage was then raised to $200{ }^{\circ} \mathrm{C}$ and maintained at that temperature for approximately $10 \mathrm{~min}$. The temperature was then lowered by suitable adjustment of the current supply to the hot stage and maintained within $\pm 1{ }^{\circ} \mathrm{C}$ of a predetermined temperature at which the rate of spherulite formation was slow enough to be recorded with a conventional photographic

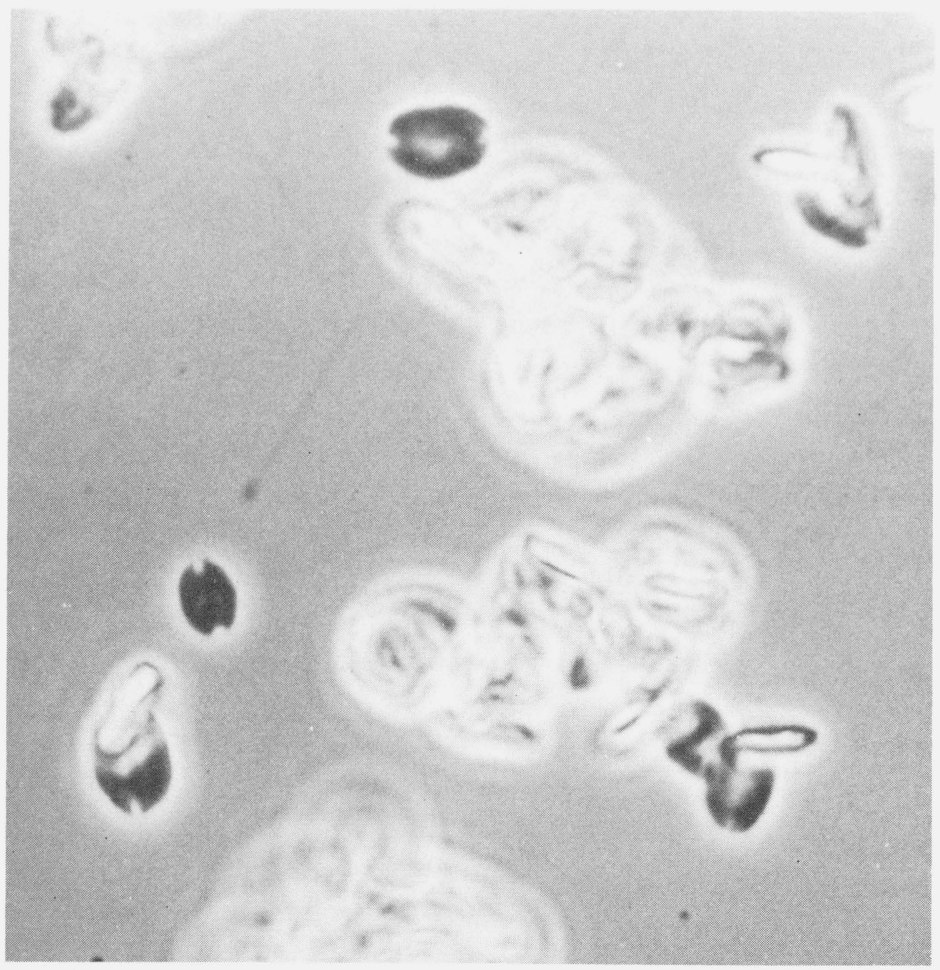

FigURE 12. General view including numerous randomly oriented aggregates from the same preparation as those shown in figures 7 to 11 . Phase Contrast, $\times 660$. 
camera. The vertical size of the heating stage and the mechanically restricted vertical travel of the phase contrast condenser system used were such that suitable illumination could be obtained only for low and medium power phase contrast objectives. All direct observations and photographic recordings were made using a $\times 20$ phase contrast objective. The sequences described below were recorded within a period of 30 to $45 \mathrm{~min}$.
A sequence of micrographs depicting successive stages of growth of an individual positively birefringent monoclinic spherulite from a 5 percent $(\mathrm{w} / \mathrm{w})$ solution of the polymer at $113{ }^{\circ} \mathrm{C}$ is shown in figure 13 (a to h). The thickness of the solution between the glass slides was of the order of $50 \mu$. The similarity between the profile of the initial successive transitional structures depicted in figure $13 \mathrm{a}, \mathrm{b}$, and that of the aggregates shown in figure $11 \mathrm{c}, \mathrm{d}$, and figure 12 is clearly evident.
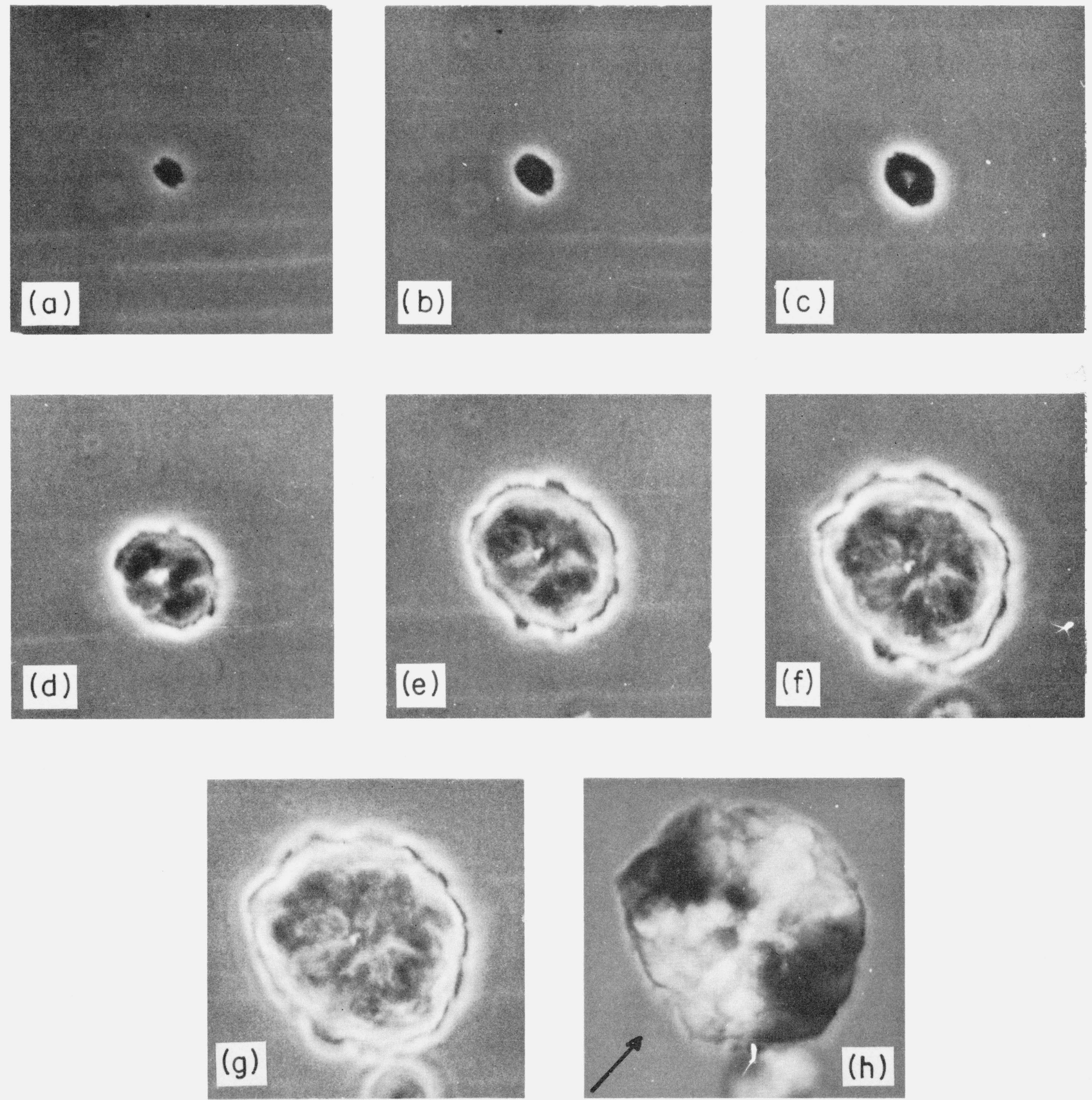

FIGURE 13. Time lapse sequence depicting the growth of a positively birefringent spherulite from a 5 percent solution of polypropylene in mineral oil at $113^{\circ} \mathrm{C}$

(a-g) Phase contrast. (h) Crossed polarizers and $1 / 4 \lambda$ compensator (arrow indicates slow direction of vibration of compensator). $\times 670$. 
Note the convex lateral edges of the successive structures shown in figure $13 \mathrm{a}, \mathrm{b}$, and the re-entrant or notched appearance these transitional aggregates exhibit at opposite extremities. This latter feature, which is less pronounced at the more advanced stage of development represented in figure $13 \mathrm{~b}$, becomes progressively less evident on subsequent growth and disappears eventually, at which stage the developing structure exhibits an essentially elliptical profile (as seen in figure $13 \mathrm{c}, \mathrm{d}$ ) which corresponds to that of the aggregates shown in figures 8 and 10 . Subsequent stages of growth culminating in the development of a monoclinic spherulite are shown in figure 13 (e to h). Figure $13 \mathrm{~h}$ represents the spherulite thus formed as seen between crossed polarizers and a quarter wave compensator revealing its positively birefringent character.

In some instances it was possible to observe and record the growth of individual spherulites at even earlier stages of development than has been depicted
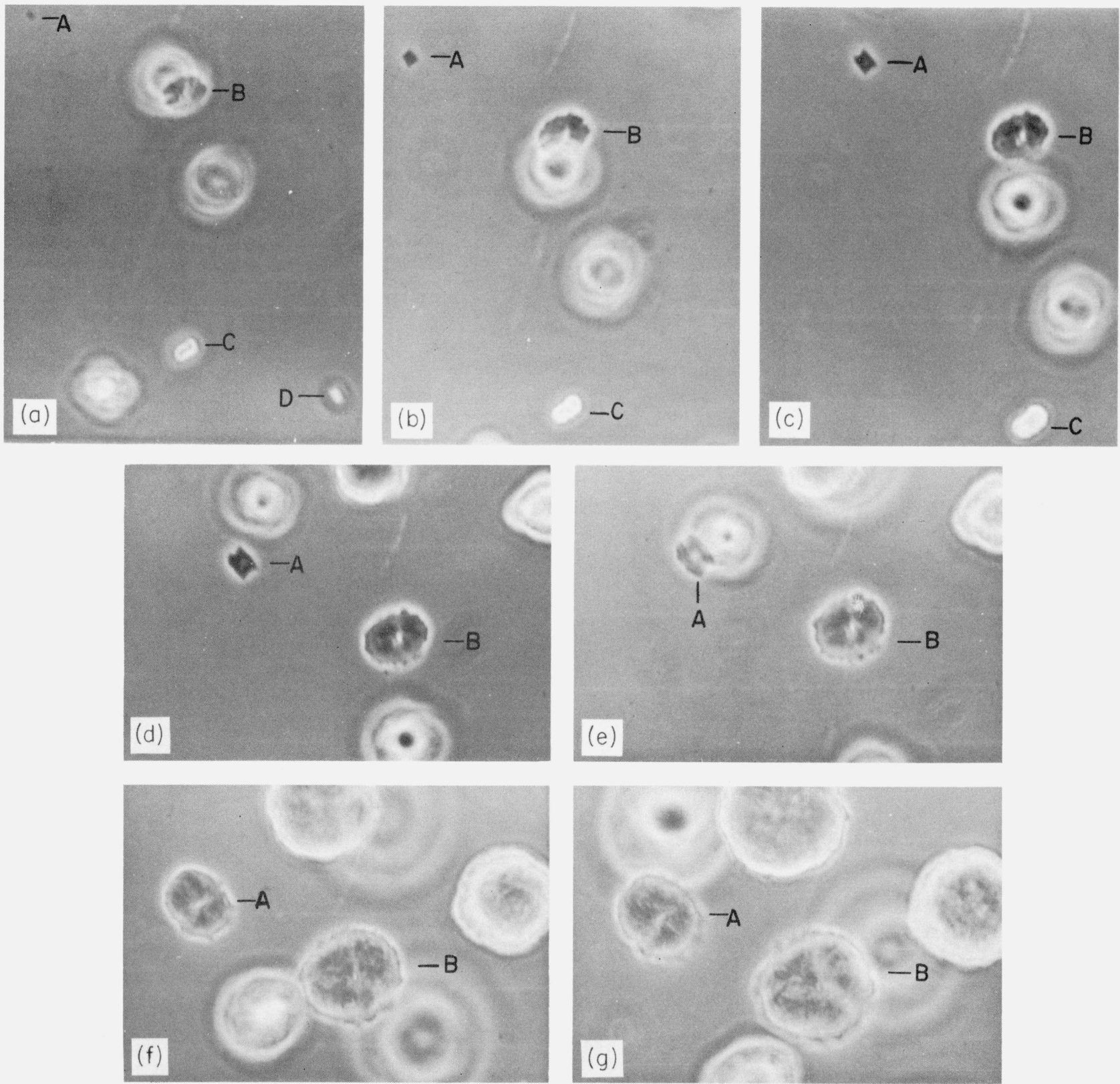

FIGURE 14. Time lapse sequence depicting successive early stages $(A)$ in the growth of a positively birefringent spherulite from a 12 percent solution of polypropylene in mineral oil at $116^{\circ} \mathrm{C}$.

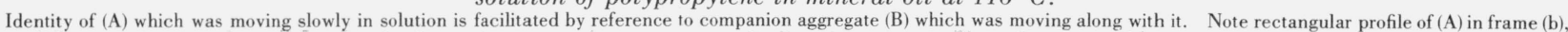
a very slight concavity of its shorter edges may be detected. See text for comments on (C), (D) in frames ( $\mathrm{a}-\mathrm{c})$. Phase Contrast. $\times 410$. 
in the sequence shown above. Correspondingly, the very initial resolvable precursor structure was found to exhibit (when appropriately oriented) a rectangular (almost square) profile. Early stages of development of an individual monoclinic spherulite from a 12 percent $(\mathrm{w} / \mathrm{w})$ solution of polypropylene in mineral oil at $116{ }^{\circ} \mathrm{C}$ are depicted in the sequence of micrographs shown in figure 14. The particular structure (A) the growth of which was followed in this sequence was moving very slowly in the solution; its identity is confirmed in figure 14 (a to g) by a reference aggregate (B), at a more advanced stage of growth, which was moving along with it (note the elliptical profile of B).

The apparently rectangular (almost square) profile of the first clearly resolvable precursor seen in figure $14 \mathrm{~b}$ is evident. At the growth stages depicted in figure $14 \mathrm{c}, \mathrm{d}$ the profile of the growing structure can be seen to correspond with that of the smallest and rarer aggregates (of the type shown in fig. 11a,b) described in the previous subsection. Note the longer lateral edges and the distinctly cusped appearance (concave with respect to the interior) of the shorter edges of the growing aggregate (A) in figure $14 \mathrm{c}, \mathrm{d}$. The aggregate subsequently assumes on further growth a profile similar to that of the structures shown in figure 11c,d. At the more advanced stages of growth depicted in figure 14 ( $\mathrm{f}$ to $\mathrm{g}$ ) the aggregate may be seen to exhibit an essentially elliptical profile-it eventually developed into a positively birefringent spherulite.

On the basis of the evident similarity between the transient precursors seen in the sequences shown in figures 13, 14, and the aggregates described in the previous subsection (3.2a), it is to be expected that their appearance would vary significantly depending on their orientation with respect to the direction of viewing, hence the contrasting appearance of precursors C (fig. 14 a to c) and D (fig. 14a). It may be readily deduced that they are respectively oriented with their plane of preferential development essentially normal to the plane of the field of view photographed in figure 14 .

In summary, the observations described in this section provide a gross insight into the nature of the early stages of development of monoclinic spherulites of polypropylene. Further opportunity to characterize in finer detail the early stages of development of such spherulites is clearly provided by the ready accessibility of the representative precursors of the spherulites formed from the $3 / 4$ percent mineral oil solutions.

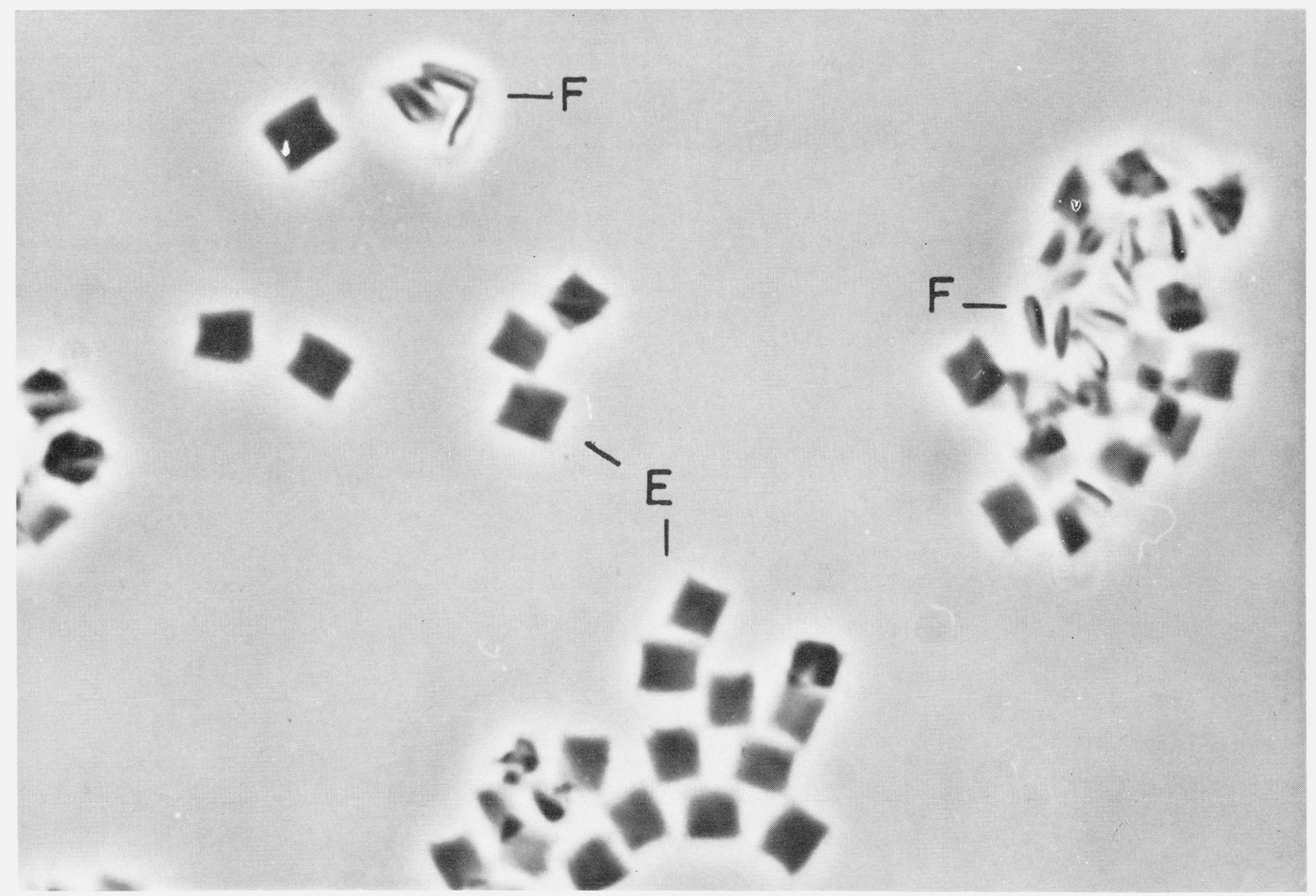

FIGURE 15. Pseudopolyhedral aggregates grown from a 0.75 percent solution of polypropylene in amyl acetate at $114{ }^{\circ} \mathrm{C}$. (E) denotes aggregates lying with their plane of preferential development parallel to the substrate. (F) denotes the same type of aggregate viewed edge-on. Note rectangular profile of aggregates (E) as well as the slightly concave nature of their shorter edges. Phase Contrast. $\times 1,400$. 
Clearly these structures can 'be readily isolated from the latter preparations and subjected to an electron microscopic study using replicating techniques in order to characterize the nature of their fine structure.

The apparently polyhedral earliest precursors which develop during the initial stages of formation of the spherulites are of particular initial interest, however. Similar essentially planar objects exhibiting a square or slightly rectangular profile have been respectively observed by Leugering [13] and Geil [14] to form during the initial stages of growth of monoclinic spherulites from the melt. In short, the formation of such initial structures is not a peculiar characteristic of the mode of growth of monoclinic spherulites under the specific conditions of crystallization described in this sectionit is evidently a phenomenon of more general occurrence. The discovery that $3 / 4$ percent solutions of polypropylene in amyl acetate yield, at temperatures between 110 to $115{ }^{\circ} \mathrm{C}$, monoclinic crystalline aggregates the shape of which (see fig. 15) coincides with that of the above-mentioned initial apparently polyhedral precursors of monoclinic polypropylene spherulites, was therefore particularly gratifying [15]. The outcome of an optical and electron microscopic study, which has led to the characterization of the highly unorthodox structure of these model counterparts of the earliest microscopically resolvable spherulite precursors, is discussed in the ensuing section. It will be shown that they are not polyhedral objects, but possess instead a complex fine structure which is distinctly dendritic in character.

\section{On the Morphology of Model Counterparts of the Pseudopolyhec'ral Precursors of Mono- clinic Spherulites of Polypropylene}

The observations presented in this section were derived from an optical and electron microscopic study of crystalline aggregates grown from $3 / 4$ percent solutions of polypropylene in amyl acetate at $114{ }^{\circ} \mathrm{C}$. Within $6 \mathrm{hr}$ at that temperature the solutions exhibit a very faint cloudiness indicating the onset of crystallization. The particular solution from which the aggregates shown in figure 15 were formed was held at $114{ }^{\circ} \mathrm{C}$ for 10 days. At the end of this period, the solvent and suspended aggregates were poured into a special filtration vessel maintained at $114^{\circ} \mathrm{C}$. The type of vessel used was essentially similar in design to a filtering apparatus previously described by Bassett et al., [16]. The preparation was filtered isothermally and washed several times with fresh amyl acetate preheated to $114{ }^{\circ} \mathrm{C}$. Care was taken not to allow the filtrate to dry between successive washings. After a final addition of hot fresh solvent, filtration was stopped, and the vessel allowed to cool to room temperature.

The characteristic monoclinic [3] wide angle $\mathrm{x}$-ray diffraction pattern (powder pattern) obtained from a sample consisting of randomly oriented aggregates formed at $114{ }^{\circ} \mathrm{C}$ is shown in figure 16 .

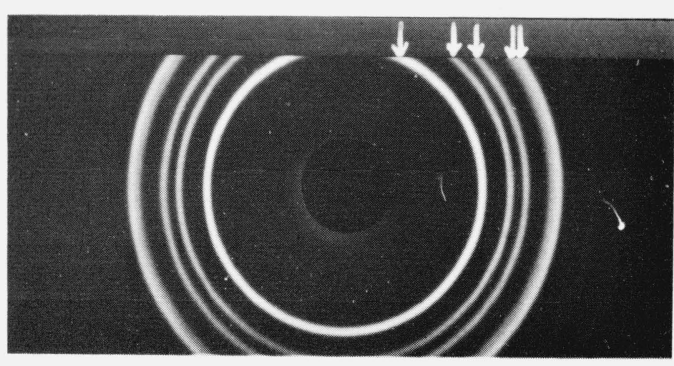

FIGURE 16. Wide angle $x$-ray powder pattern from a sample consisting of pseudopolyhedral structures of the type shown in figure 15.

The inner 110,040, 130 and composite 111, 131, 041, monoclinic polypropylene rings are indicated by vertical bars.

\subsection{Observations Derived From Optical (Phase Contrast) Microscopy}

Examination of the aggregates formed in the preparation described above at high magnifications under the phase contrast microscope as they tumbled in suspension in liquid revealed that they possess a planar habit, and that when viewed along an axis normal to their plane of preferential development (e.g., the aggregates marked (E) in fig. 15) they exhibit an essentially rectangular (almost square) profile. Characteristically however, the shorter of the two pairs of lateral edges appear either slightly cusped near the apices or are slightly curved, concave with respect to the interior of the aggregate. The length of the longer pair of lateral edges was generally in the range 5 to $7 \mu$. The thickness of these structures along the axis normal to their plane of preferential development, i.e., along the direction normal to the cross section whose peripheral characteristics have been just described, is of the order of 1 to $2 \mu$. When viewed edge-on as is the case of aggregates marked (F) in figure 15, they exhibit a rodlike or blunt spindlelike appearance.

In so far as one could ascertain under the optical microscope the aggregates appeared to possess substantial overall rigidity. No detectable changes in profile were observed under the phase contrast microscope when they were allowed to dry in air at room temperature. This feature is further confirmed by the electron microscopic observations presented below and will be discussed in a later part of the paper.

\subsection{Observations Derived From Electron Microscopy}

\section{a. Direct Examination in Transmission}

The aggregates were deposited on electron microscope grids covered with a carbon film and examined directly in the electron microscope. It became immediately evident that they were highly susceptible to the electron beam. At levels of illumination that were high enough to permit clear visual examination of the image formed on the fluorescent screen of the microscope, the aggregates were invariably observed to shrivel up extremely rapidly. This drawback was 
overcome by scanning the grids and photographing the aggregates at very low intensities and by limiting the area of the specimen grid exposed to the electron beam at any given time to approximately one grid square. This was accomplished by the conventional method of using the double condenser system of the microscope and appropriately defocusing the second condenser lens.

Under such conditions of low illumination it was possible to discern only faintly the outline of individual aggregates on the microscope screen. Consecutive micrographs recorded within $30 \mathrm{sec}$ of exposure of individual aggregates to the electron beam under such conditions of low "illumination" revealed no detectable morphological changes in that period of time. Within two minutes, however, substantial changes in overall shape were discerned, even visually.

Electron micrographs depicting three individual aggregates photographed immediately after being exposed to the electron beam are shown in figures 17, 18, and 19a. These aggregates originate from the same preparation as the structures shown in figure 15 . Two characteristic features detected under the optical microscope are clearly revealed in these electron micrographs, namely, the overall essentially rectangular profile of each aggregate and the slightly cusped or concave nature of the shorter "sides." The length to breadth ratio of the aggregates (as determined from the length of the central axes bisecting opposite sides) was generally found to be of the order of 1.1:1.

As would be expected from the 1 to $2 \mu$ thickness of these structures they are essentially opaque to the electron beam, particularly so in the somewhat thicker central regions. Nevertheless, transmission electron micrographs generally revealed two characteristic morphological features. First, what appeared to be narrow diagonally oriented spikelike outgrowths were frequently seen protruding from the edges of the aggregates, as may be seen in particular in figures 17 and 19a. Second, a weakly discernible diagonally oriented cross-hatched appearance which could be traced from the peripheral regions for a limited distance into the interior, as may be seen for example in figure 18, was frequently detected in the electron micrographs.

In addition to the above-mentioned features apparently spurious lamellar single crystallike outgrowths, as may be seen for example near the apex $(G)$ of the aggregate partially in view in figure 18, were occasionally observed in the peripheral regions of some aggregates.

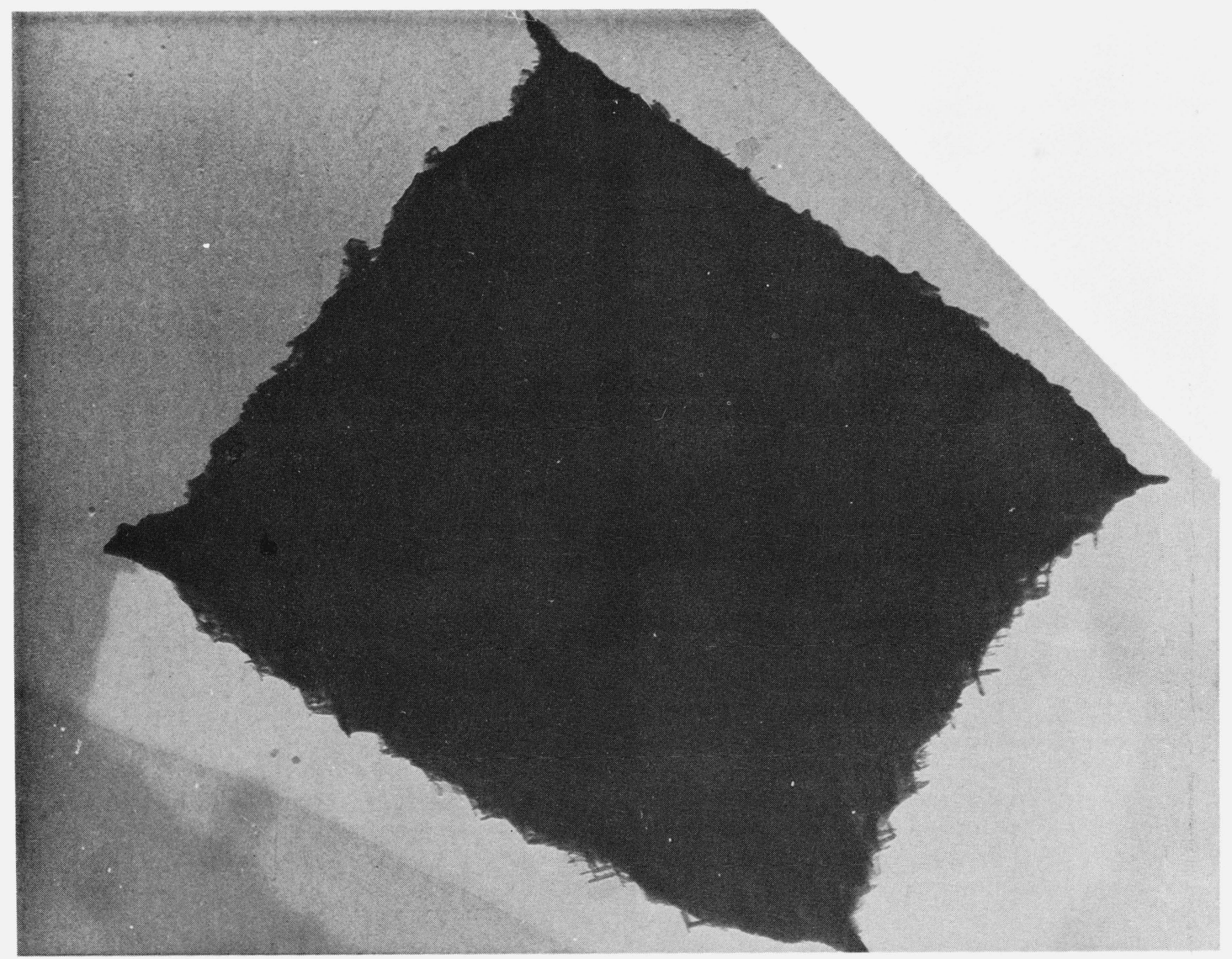

FIGURE 17. Electron micrograph of a pseudopolyhedral polypropylene aggregate of the type shown in figure 15.

Note overall essentially rectangular profile of the aggregate as well as the diagonally oriented spikelike outgrowths protruding from its periphery. Also note slightly concave profile of one of its shorter "sides." Shadowed (Pt.-Pd). $\times 16.800$ 
The consequence of prolonged exposure to the electron beam is shown in figure 19b. The aggregates were always observed to undergo preferential overall shrinkage along a direction normal to their longer axis as may be readily ascertained by comparing figure $19 \mathrm{a}$ and figure 19b. No further change in aspect was observed beyond the stage of shriveling depicted in the latter electron micrograph even when the intensity of illumination is substantially increased. The specificity of the mode in which the aggregates deform remains unexplained. The change they undergo when exposed to the electron beam is primarily attributable to melting or partial melting resulting from the heating effects of the electron beam and the poor dissipation of heat generated by the impinging electrons due to the low thermal conductivity of the aggregates and their supporting carbon film. It is to be expected that the polypropylene chains undergo cross-linking on exposure to the electron beam [17]. On the basis of established experience, for example contrasting the behavior of these aggregates with that of polyethylene single crystals, it may be readily assumed that the occurrence of cross-linking (which leads to a loss of crystallinity in polyethylene crystals without their undergoing a change in shape) is not the prime factor leading to the drastic deformation which the aggregates undergo when irradiated.

\section{b. Electron Microscopic Examination of Surface Replicas}

In order further to elucidate the nature of the fine structure of the aggregates, replicas of their large surfaces were prepared using the method of shadow transfer. For this purpose, the aggregates were initially deposited on a glass slide and allowed to dry in air at room temperature; they were then shadowed with metal (80 percent $\mathrm{Pt}-20$ percent $\mathrm{Pd}$ alloy). A backing film of carbon was subsequently evaporated onto the shadowed aggregates on the slide. The resulting composite metal-carbon "film" thus formed was floated over distilled water. The aggregates were found to adhere to the film, portions of which

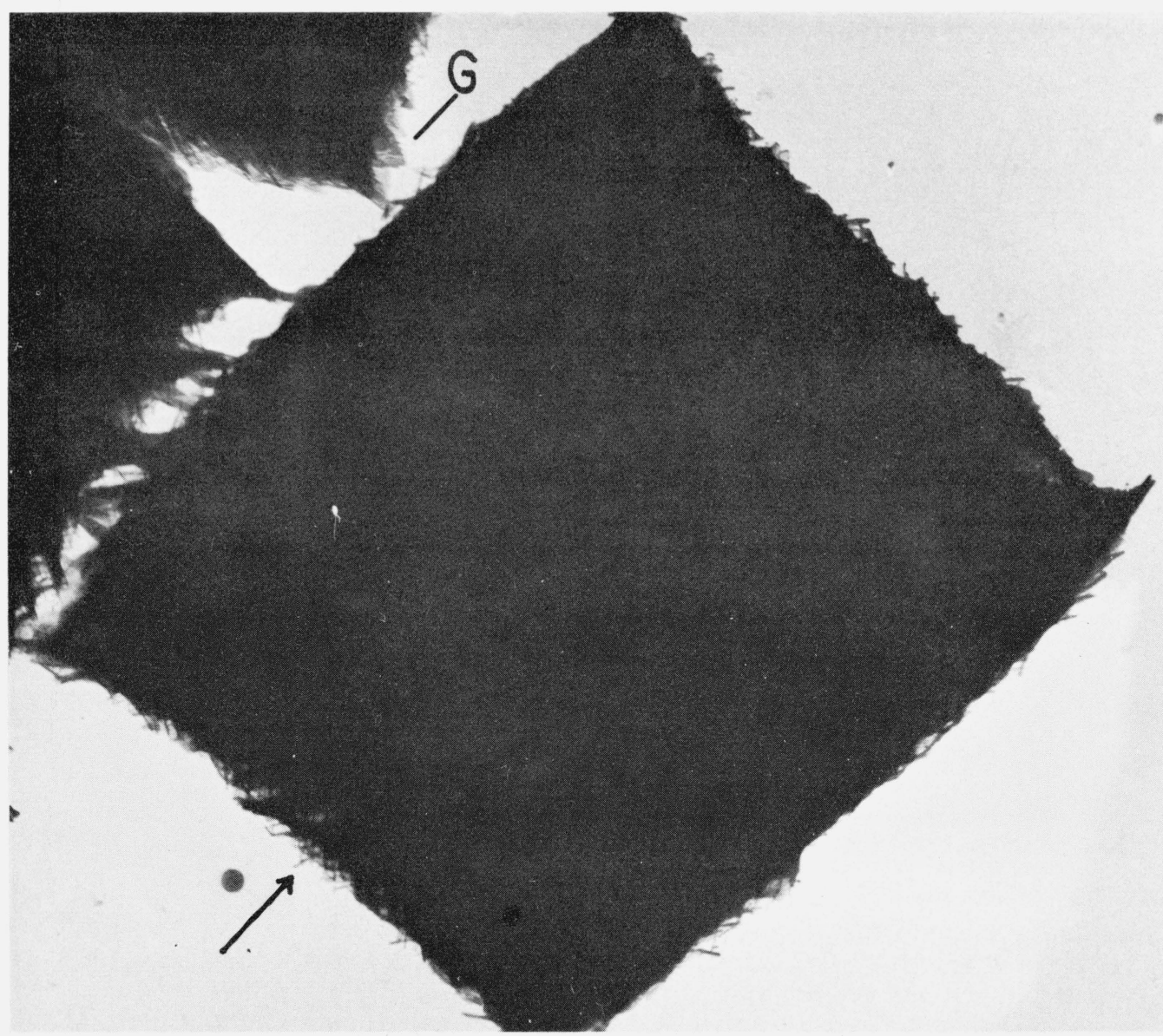

FiguRE 18. Electron micrograph of a pseudopolyhedral polypropylene aggregate.

Note the faintly discernible diagonally oriented cross-hatched appearance extending inwards from one of the shorter edges of the aggregate indicated by the arrow near the left-hand corner of the figure. Also note apparent lamellar character of outgrowths (G) near the apex of another neighboring aggregate. Shadowed Pt-Pd $\times 16,400$. 

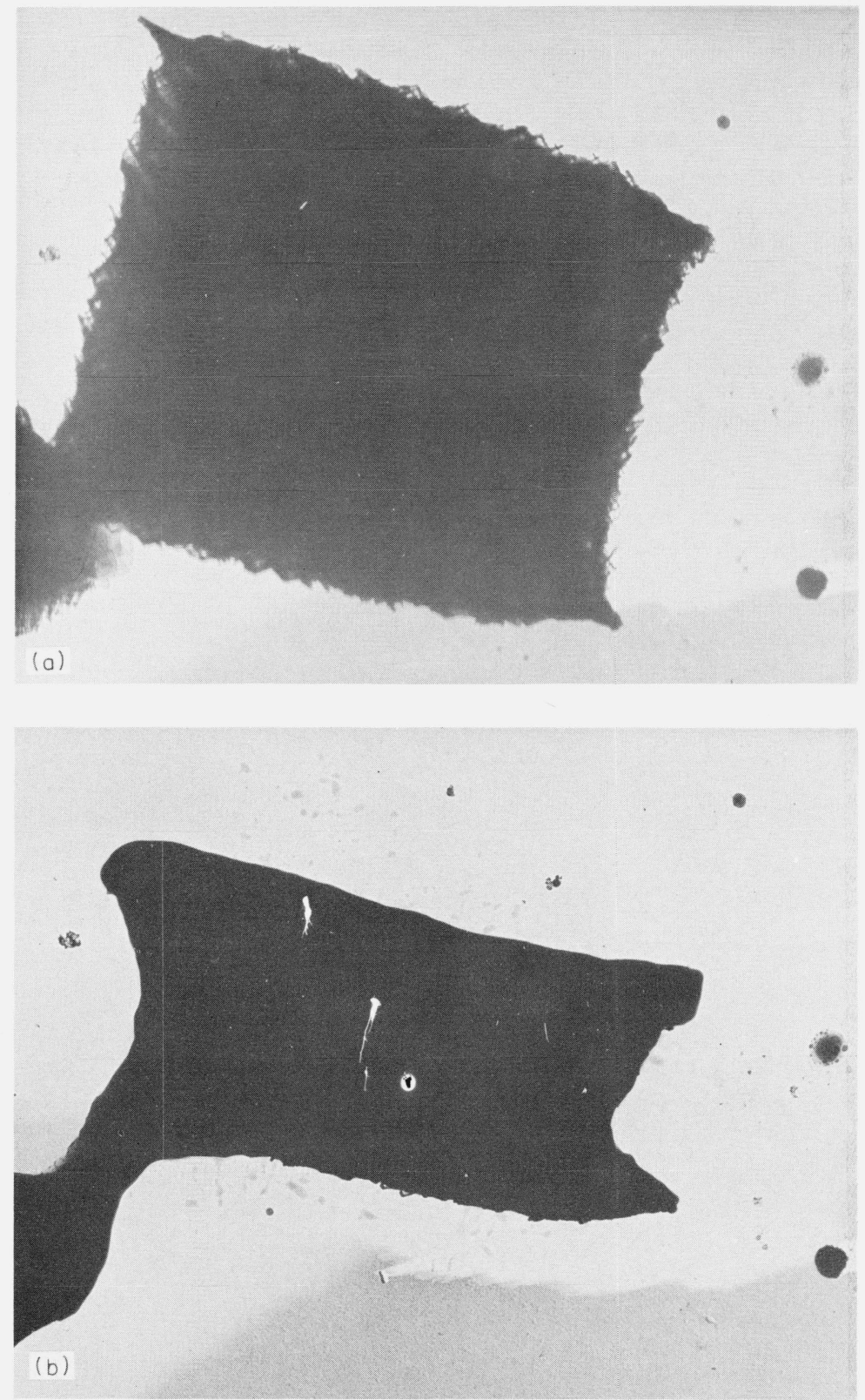

FIGURE 19. Electron micrographs depicting effect of prolonged exposure to the electron beam.

(a) Micrograph recorded at low intensity immediately after exposure to the electron beam. (b) Micrograph of same aggregate after $2 \mathrm{~min}$ exposure to the electron beam. Note characteristic preferential shrinkage at right angles to the longer edges of the aggregate. Shadowed $(\mathrm{Pt}-\mathrm{Pd}) \times 13,300$.

were then picked up on electron microscope grids and allowed to dry in air at room temperature. Some difficulty was encountered in dissolving the aggregates from the metal-carbon replicating medium but they were effectively dissolved by immersing the replicas supported on the grids in excess distilled decalin, heating the solvent to $160{ }^{\circ} \mathrm{C}$, and maintaining it at that temperature under a blanket of nitrogen for $18 \mathrm{hr}$.

Replicas of the large aggregate surfaces prepared in the manner described above revealed, as may be seen in figure 20 , a distinct cross-hatched surface texture consisting of a network of narrow (150 to $200 \AA$ ) apparently intercrossing structural units which traverse the surface for varying distances diagonally 


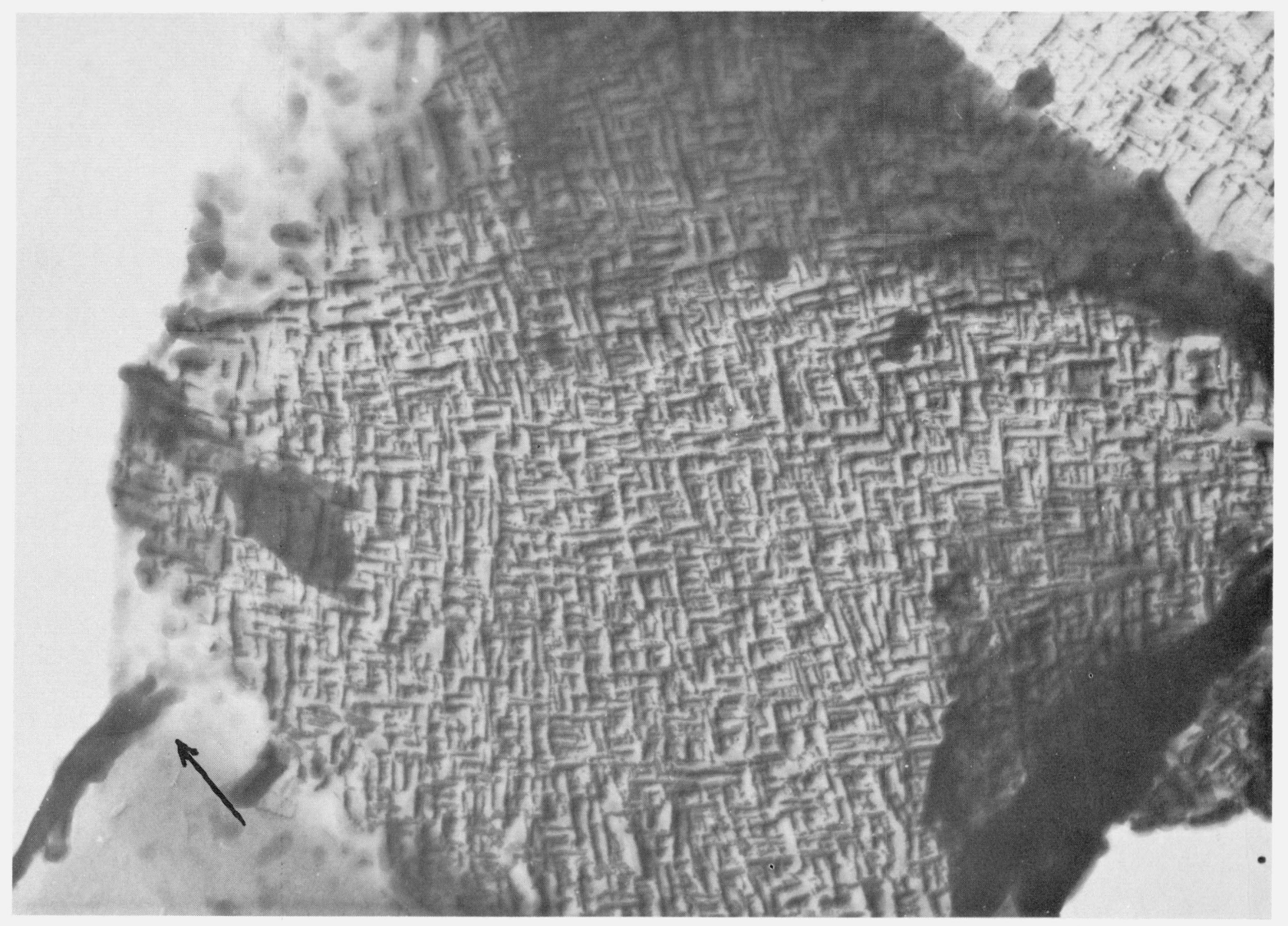

FIGURE 20. Electron micrograph of a shadow transfer replica of the surface of a pseudopolyhedral polypropylene aggregate.

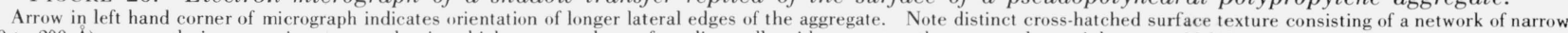
$(150$ to $200 \AA$ ) apparently intercrossing structural units which traverse the surface diagonally with respect to the rectangular periphery. $\times 24.100$.

with respect to the aggregate periphery. A magnified view of the central portion of the replica depicted in figure 20 is shown in figure 21 in which the dense but not compact network of narrow diagonally oriented structural units is clearly seen.

In summary, the preliminary morphological observations derived from the examination of the aggregates in transmission and by replication clearly established that they possess a composite fine structure having the earmarks of a crystallographically specific pattern of growth which is dendritic in character-the narrow diagonally oriented structural units being readily identifiable as elementary (primary, secondary, etc.) dendrite branches.

On the basis of the foregoing morphological observations several questions immediately arose, inter alia: How is growth propagated normal to the plane of preferential development of the aggregates? What is the nature of the molecular organization in the constituent branches? A possible clue relating to the nature of both these aspects was provided by the occasionally observed lamellar, and hence one could reasonably presume chain-folded, single crystallike peripheral outgrowths revealed, as mentioned earlier, in transmission electron micrographs of some aggregates. The apparently spurious nature of these lamellar outgrowths seemed inconsistent with the highly organized fine structure revealed by the surface replicas (in particular) which evidently reflected a highly specific mechanism of growth. This consideration led to the conjecture that these lamellae might simply be individual branches of the type revealed by the replicas (the narrow diagonally oriented structural units pointed out in figs. 20 and 21) which, however, were being seen in a different perspective as a consequence of localized distortions undergone inadvertently by the aggregates in their peripheral regions on being transferred to, or during subsequent drying on, the electron microscope sample grids.

In short, the possibility was considered that the narrow diagonally oriented branches revealed by the replicas are lamellar chain-folded single crystallike structures whose fold surfaces are normal or preferentially normal to the aggregate plane of preferential development. Clearly, this would readily account for the apparent uniform width of the branches when: viewed along that same direction. Substantiation of the foregoing conjectures is presented below. 


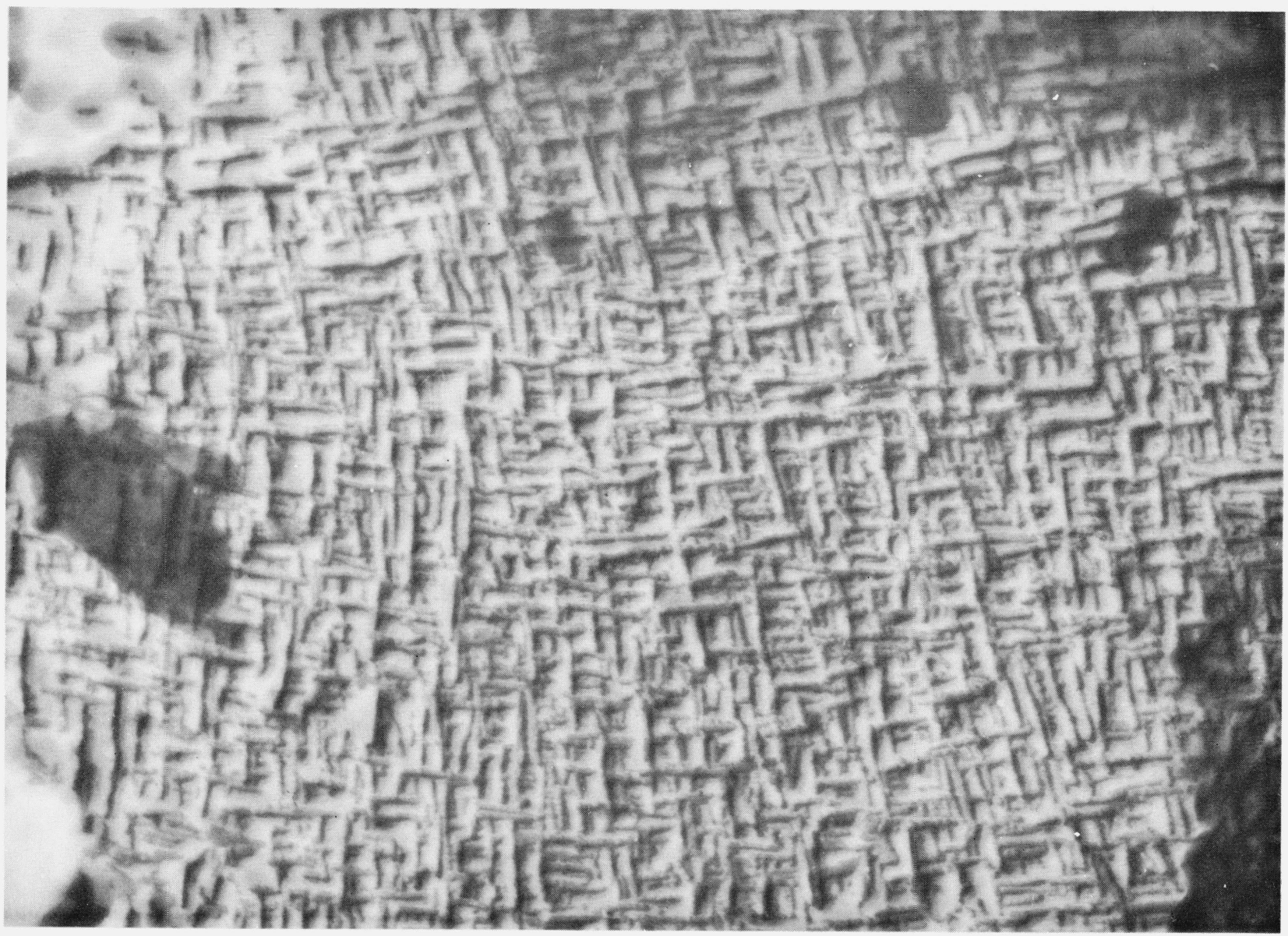

FIGURE 21. Magnified view of central portion of replica shown in figure 20. Note dense but not compact network of intercrossing structural units. $\times 39000$.

\section{c. Morphological Observations on the Pseudopolyhedral Spherulite Precursors Isolated From the Mother Liquor at an Early Stage of Growth}

Further elucidation of the nature of the internal structure and mechanism of growth of the aggregates discussed in the previous two subsections was provided by isolating such aggregates from the mother liquor at a relatively early stage in their growth and examining the fine structure of these smaller (and hence more electron transparent) precursors electronmicroscopically in transmission.

Examination under the phase contrast microscope of aliquot samples removed periodically from a $3 / 4$ percent solution of polypropylene in amyl acetate from which the polymer was crystallizing at $114{ }^{\circ} \mathrm{C}$, revealed that after a period of approximately $9 \mathrm{hr}$ at that temperature, the average size of the aggregates formed was approximately half that of those discussed in the preceding subsections. At this point the solution was filtered isothermally in the manner described earlier. Fresh solvent preheated to $114{ }^{\circ} \mathrm{C}$ was added intermittently to the preparation during filtration to wash away polymer still in solution. Care was taken not to allow the residue to dry between successive additions of fresh solvent in order to prevent the formation of a compact mat of aggregates on the filter which might be difficult to redisperse. Inevitably however, the aggregates were repeatedly drawn together during the process of filtration thus giving rise to a high incidence of random interaggregate impingement and subsequent adherence as a consequence of superficial intergrowing at regions of contact particularly during the early stages of filtration.

The intergrowing and consequent adherence of groups of randomly oriented aggregates as rigid clusters which remained as such after transfer and drying on the carbon coated electron microscope grids proved to be highly beneficial. It provided, as demonstrated below, a means of examining aggregates in widely different perspectives ranging from "normal" to edge-on viewing; ${ }^{7}$ clearly such widely divergent viewings, equivalent to tilting an individual aggregate through $90^{\circ}$ could not have been easily accomplished otherwise in the electron microscope.

i.e., along directions normal to their plane of preferential development and along directions parallel to their plane of preferential development respectively. 


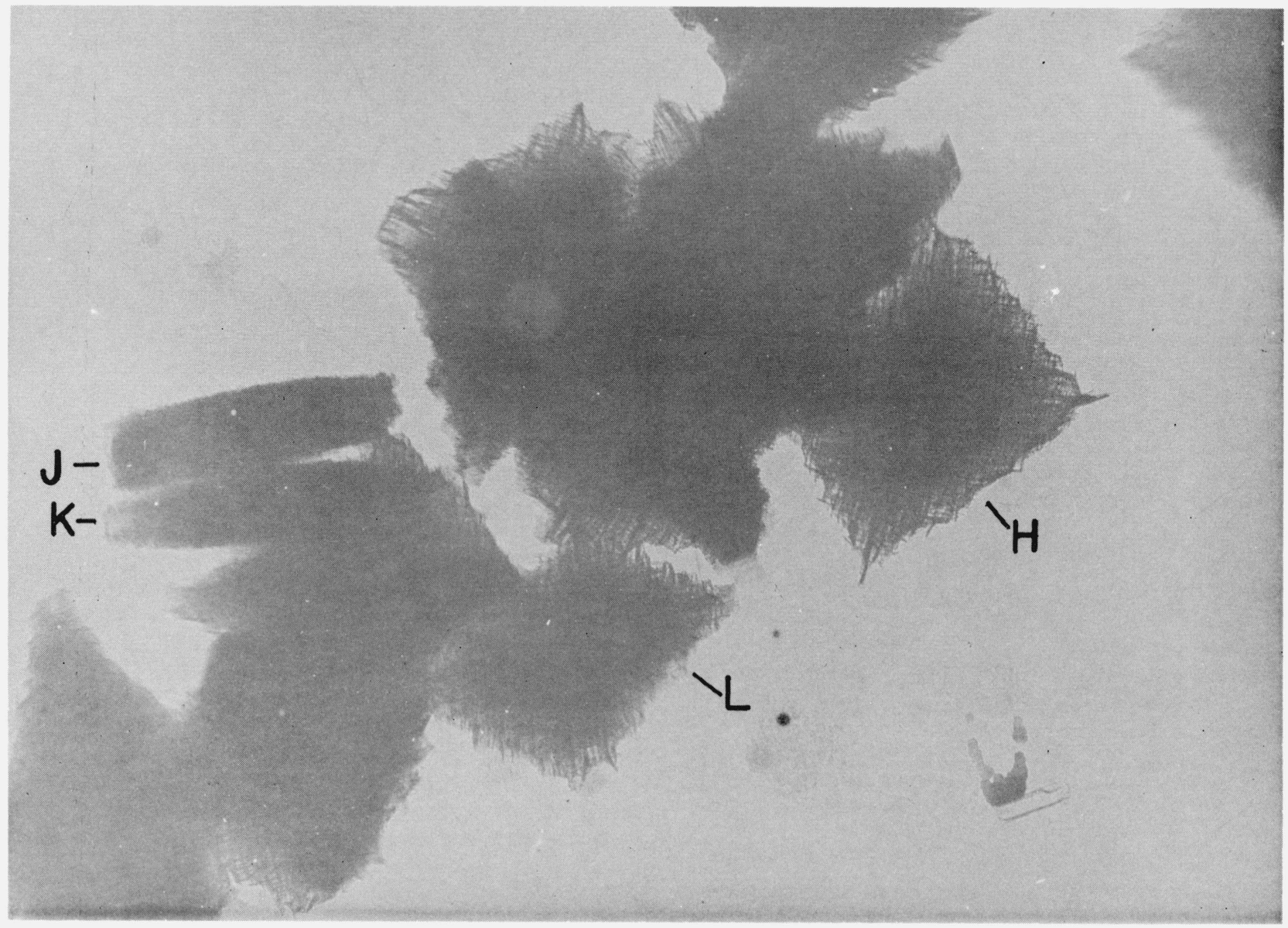

FIGURE 22. Electron micrograph of a cluster of variously oriented "half-grown" pseudopolyhedral polypropylene aggregates. $(\mathrm{H})$ is oriented with its plane of preferential development at right angles to the microscope axis, $(\mathrm{J})$ and $(\mathrm{K})$ are viewed edgè-on, and $(\mathrm{L})$ is an aggregate at an inclination intermediate between the extreme orientations represented by $(\mathrm{H}),(\mathrm{J})$ and $(\mathrm{K}) . \times 12,800$.

An electron micrograph depicting a cluster of "half-grown" aggregates isolated from the mother liqour at $114{ }^{\circ} \mathrm{C}$ in the manner described above, is shown in figure 22 . Characteristically the aggregates are variously oriented; in particular, aggregate $(\mathrm{H})$ (note its rectangular profile) is oriented "normally" with respect to the microscope axis, whereas aggregates $(J)$ and $(K)$ are oriented on edge. The plane of aggregate $(\mathrm{L})$ is inclined to the substrate. It should be pointed out that these "half-grown" aggregates were found also to be susceptible to the heating effects of the electron beam, hence the precautions described earlier (i.e., scanning and photographing at low beam intensities and suitably limiting the area of a specimen grid exposed to the electron beam at any time) had to be resorted to. The characteristic morphological features exhibited by the aggregates when viewed "normally" and edge-on are considered in detail below.

An examination of electron micrographs of aggregates oriented with their plane at right angles to the electron beam revealed the following structural characteristics which may be readily discerned in the representative aggregate shown in figure 23 . First, they exhibit an overall rectangular (almost square) profile, their length to breadth ratio being of the order of $1.1: 1$. On average they were found to be about $2.5 \mu$ long. Second, they exhibit a highly regular fine structure consisting of a dense but not compact "network" of narrow diagonally oriented branches. This evidently dendritic cross-hatched fine structure could in all instances be traced throughout each aggregate thus oriented from the thicker central regions to the periphery. The width of the constituent branches (based on the measurement of those branches that are individually resolvable in the peripheral regions) is of the order of 150 to $200 \AA$.

On the basis of geometric considerations (length/ breadth ratio), the acute angle subtended at the center of the crystals by the diagonally oriented branches (i.e., the angle subtended at the center by the shorter aggregate edges) is estimated to be $\sim 85^{\circ}$. Accurate direct interbranch angular measurements are frustrated by the overall opacity of the crystals which are $0.5 \mu-0.75 \mu$ thick.

These observations further confirmed that the crosshatched surface structure revealed by the replicas of the larger precursors described earlier is character- 


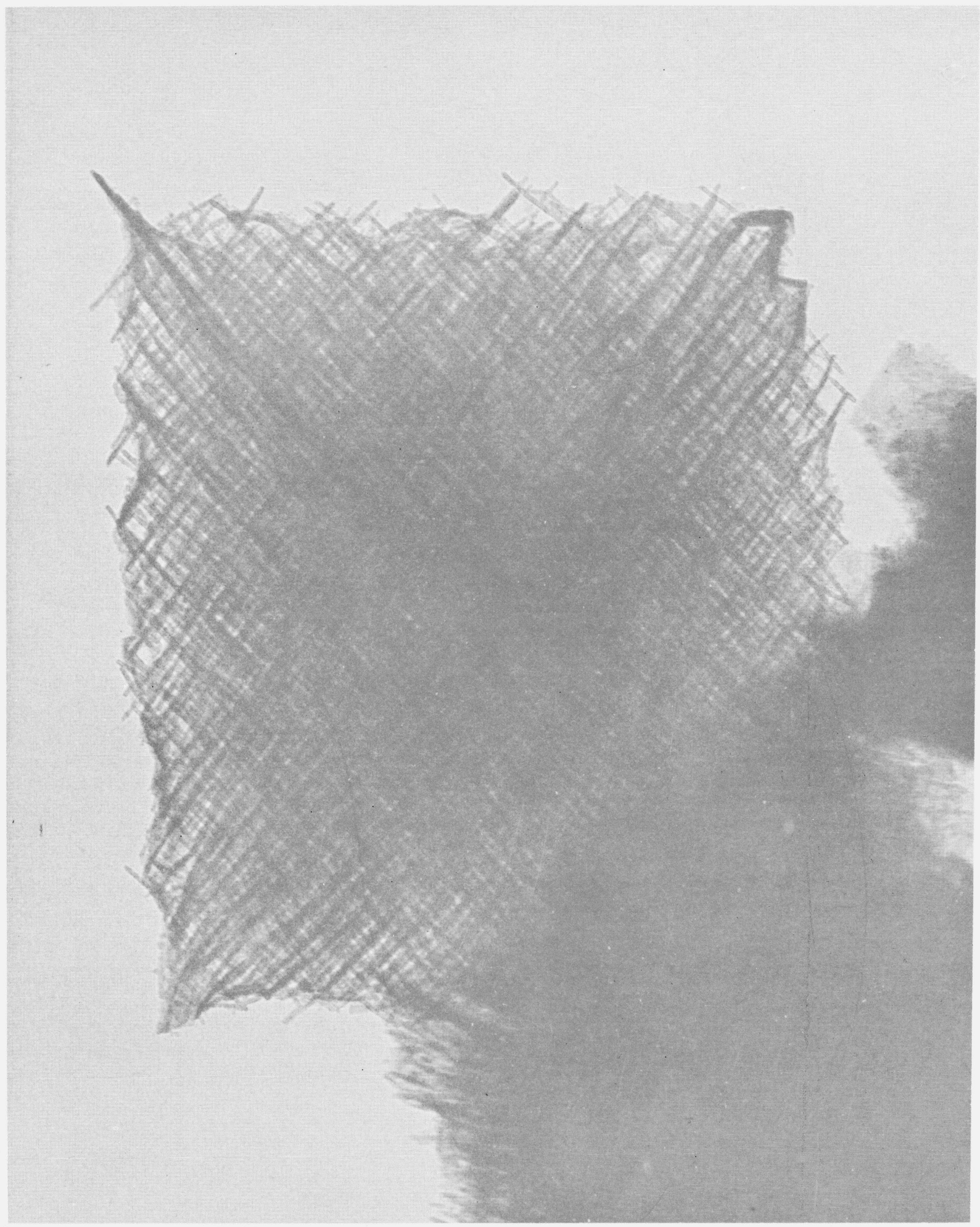

FIGURE 23. Electron micrograph revealing the distinctly dendritic crystal character of the polypropylene aggregates grown from 0.75 percent solutions of polypropylene in amyl acetate at $114^{\circ} \mathrm{C}$.

Note rectangular overall profile of the crystal as well as network of narrow diagonally oriented branches. $\times 49,900$. 


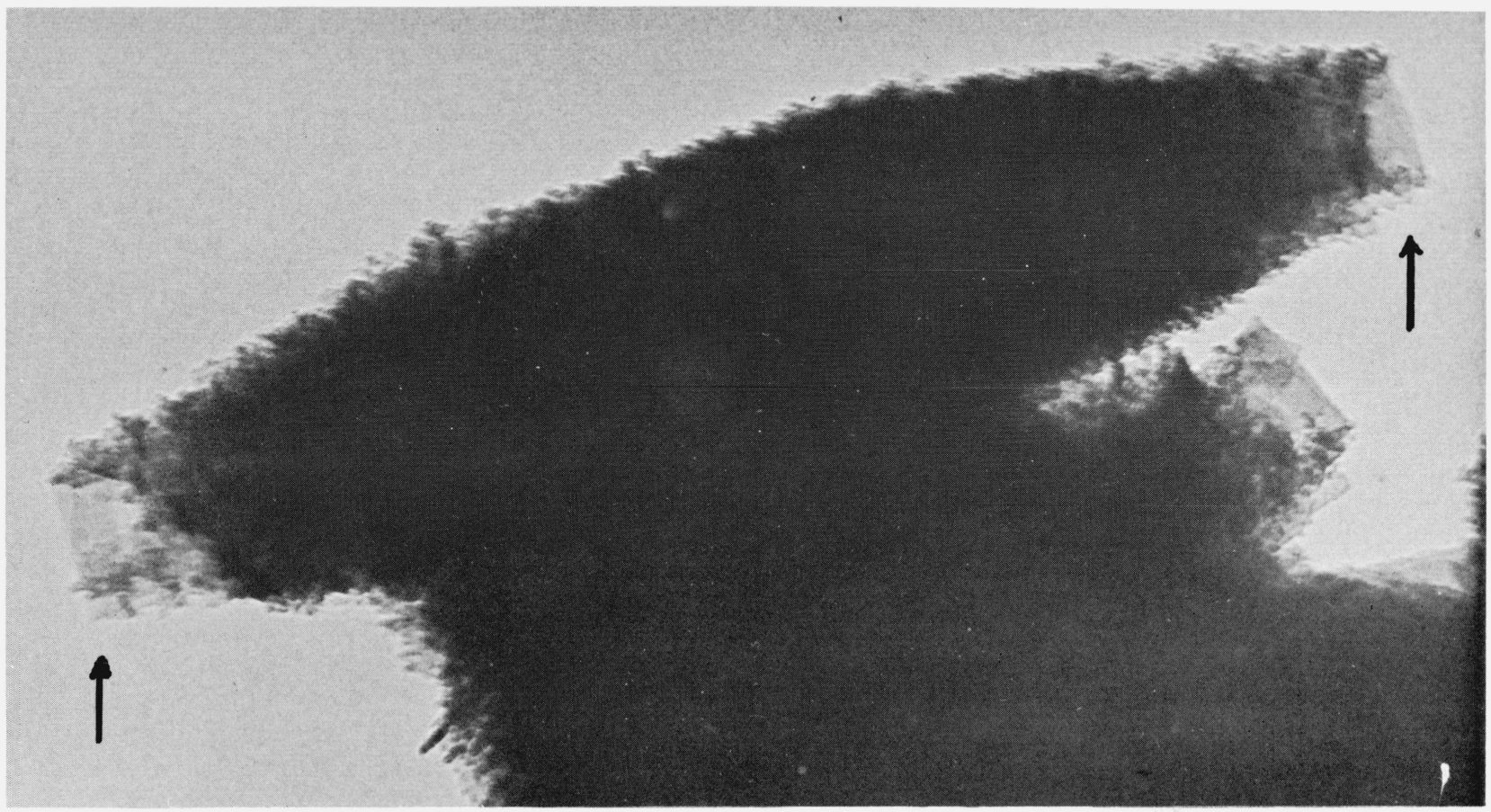

FIGURE 24. Electron micrograph of polypropylene dendrite similar to that shown in figure 23 but viewed edge-on. Note cigar shaped profile of the dendrite as well as lamellar outgrowths (arrows) protruding at both extremities. $\times 53,000$.

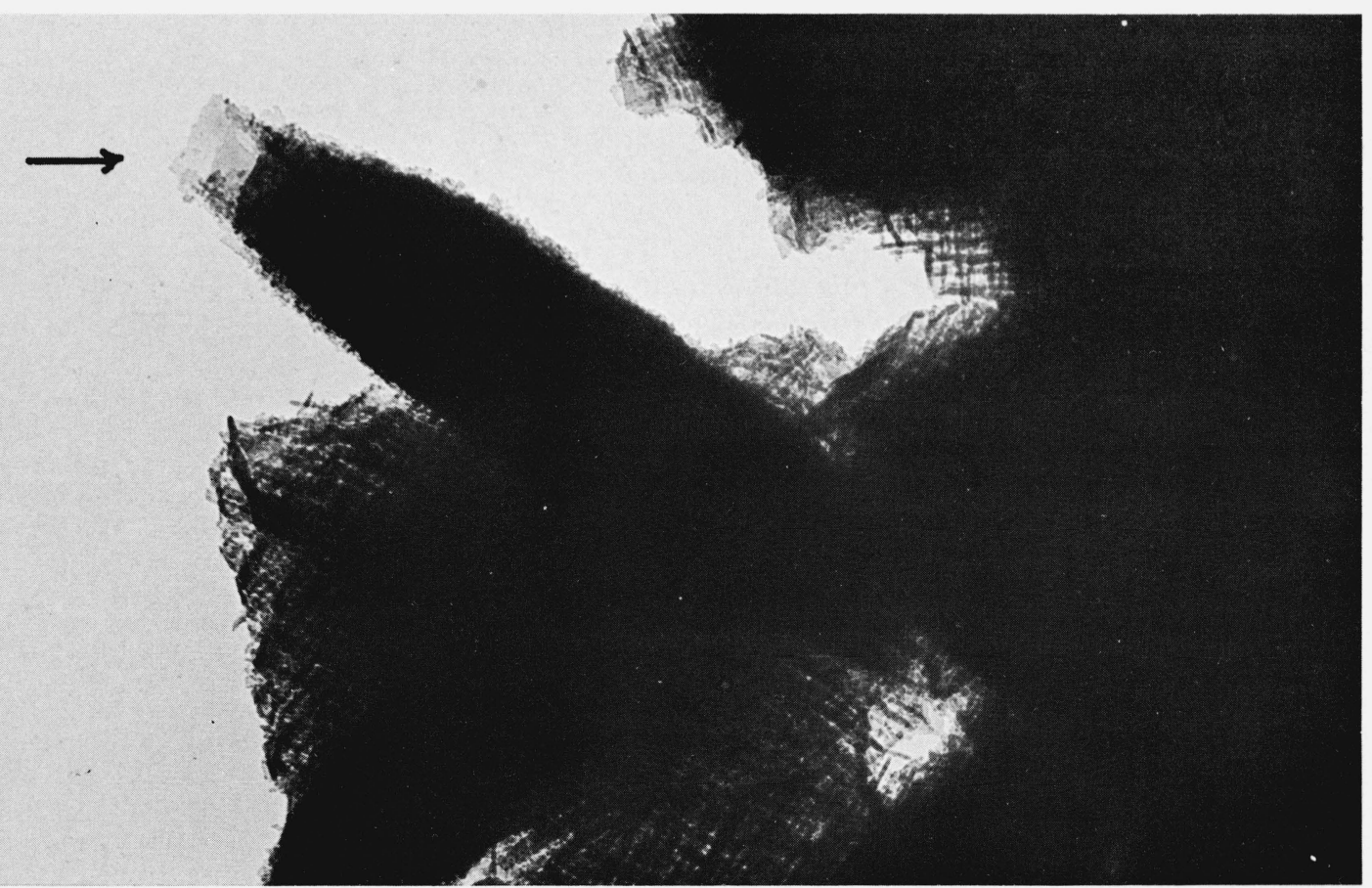

FIGURE 25. Cluster of dendrites from same preparation as those shown in figures 22 to 24. Note lamellar outgrowths (arrow) at extremity of dendrite viewed edge-on. $\times 26,100$. 
istic of their internal structure. The continuity of the same dendritic pattern of branch propagation parallel to the plane of preferential development of the smaller precursors just described and the larger aggregates discussed in the previous subsections is evident. Some loss of specificity in the mode of propagation of the branches as these dendrites become larger is however suggested by regional deviations in parallelism of the branches revealed by the replicas of the bigger dendrites and by the apparent concavity of their shorter "sides."

We turn now to a consideration of how these evidently dendritic crystals propagate along the direction normal to their plane of preferential development. In this respect the structural features exhibited by the smaller "half-grown" dendrites when viewed edge-on (as exemplified in fig. 22 (structures $\mathrm{J}, \mathrm{K}$ ) and figs. $24,25)$ were examined. When viewed thus, i.e., along directions parallel to their plane of preferential development, they exhibit a cigar-shaped profile; they were on average found to be $0.75 \mu$ wide at the center and somewhat narrower at the extremities, which is consistent with the observed decrease in overall opacity to the electron beam from the central to the peripheral regions when they are viewed as shown in figure $22(\mathrm{H})$ and figure 23 . In all instances in which the dendrites were examined edge-on, distinctly lamellar single crystallike outgrowths were observed protruding from their extremities as may be seen in figures 24 and 25; further the profile of these lamellae always formed a natural extension of the overall cigar-shaped contour of the dendrites (see both extremities of the dendrite shown in fig. 24 and the one visible extremity of the dendrite shown in fig. 25). This latter feature, coupled with the general observation that no such lamellae were seen protruding elsewhere in dendrites examined in edge-on perspective indicated that the lamellae are not spurious outgrowths but are the extremities of constituent diagonally oriented branches which extend from the interior to the rectangular periphery of the crystals.

The evidence presented in the preceding paragraph substantiates the proposition advanced earlier that the uniformly narrow diagonally oriented dendrite branches are chain-folded crystal entities whose fold surfaces are oriented at right angles to the dendrite plane of preferential development. These branches once formed can be readily visualized to develop in a continuous fashion at right angles to that plane as well as diagonally during the growth process. A simplified and essentially self-explanatory schematic representation of the mode of growth of these polypropylene dendrites as presently envisaged is shown in figure 26. For the sake of simplicity only four primary and two secondary branches are depicted; their respective fold surfaces (f) (i.e., the faces between which the molecules fold back and forth) are also identified. Clearly, profuse crystallographically specific branching involving a dihedral angle of $\sim 85^{\circ}$ between the fold surfaces in parent and offspring branches is envisaged.

The apparent overall rigidity of the dendrites may be attributed to interlocking at points where branches impinge and grow "around" one another as depicted in figure 26.

It should be emphasized at this stage that the pattern of development exhibited by these dendritic crystals is highly unusual. It is common experience that solution-grown chain-folded polymer crystals (lamellae) generally thicken along a direction normal to their fold surfaces through the agency of screw dislocations (with Burger's vectors parallel to the chain segments). These dislocations give rise to the formation of additional chain-folded terraces which are coplanar with the basal lamella. ${ }^{8} \quad$ As far as this author is aware, no previous instances have been reported, in the context of chain-folded crystals of other polymers, of the occurrence of the repeated formation of lamellar outgrowths the fold surfaces of which are oriented at a substantial and specific angle $\left(\sim 85^{\circ}\right.$ in the present instance) to the fold surfaces of the parent lamellae from which they have grown, as exemplified by the polypropylene crystals described herein.

Further elucidation of the structure and mechanism of growth of these unusual dendritic crystals clearly required their characterization on a crystallographic basis. Some observations pertaining to the nature of the molecular orientation in the dendrites are presented below.

\section{d. On the Crystallography of the Dendrites}

The birefringent properties of the dendrites were determined by examining the crystals orthoscopically under a polarizing microscope while they were in suspension in amyl acetate and other liquid media at room temperature. The resulting observations are described below and their significance in so far as providing a rough indication of the nature of the molecular orientation in the dendrite branches is discussed in conjunction with some limited electron diffraction data. Electron diffraction experiments have been somewhat frustrated by the instability of the crystals in the electron beam, as well as by the overall opacity of the crystals to the impinging electrons. In a few instances the crystals were preserved long enough at low illuminations that a selected area electron diffraction pattern as well as the corresponding selected area including a crystal were recorded photographically. The few diffraction patterns which have so far been recorded, and which will be discussed presently, were extremely weak; they could not be discerned visually on the fluorescent screen of the electron microscope. Evidently only the peripheral regions of the dendrites contributed to the diffraction patterns.

The room temperature extinction characteristics of the larger dendrites (such as those shown in figs. 15, 17 to 21), when variously oriented with respect to crossed polarizers as well as the corresponding inter-

\footnotetext{
${ }^{8}$ The situation is somewhat more complex in the case of hollow pyramidal crystals. In such instances the additional layers are also hollow pyramidal and coaxial with the "basal" such instaner [18].
laye
} 


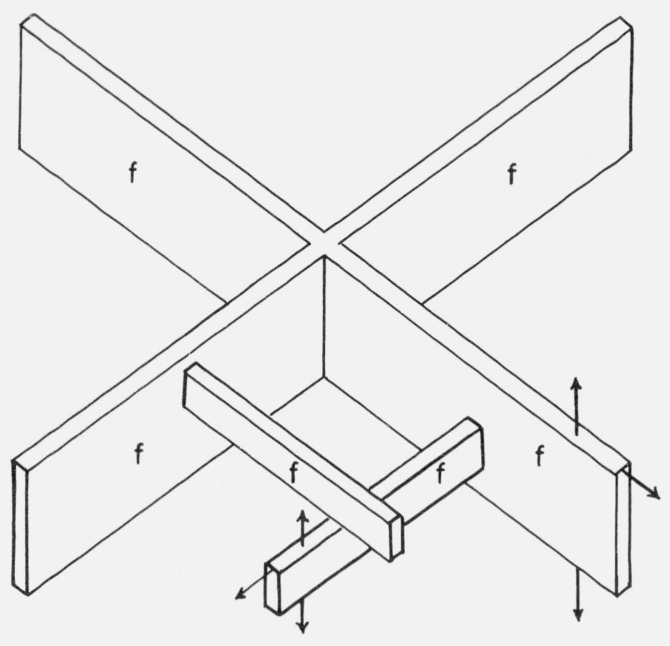

FIGURE 26. Simplified schematic representation of the structure and mode of branch propagation of the polypropylene dendrites described in figures 15 to 25.

The faces between which the chain molecules in individual branches fold back and forth are denoted (f).

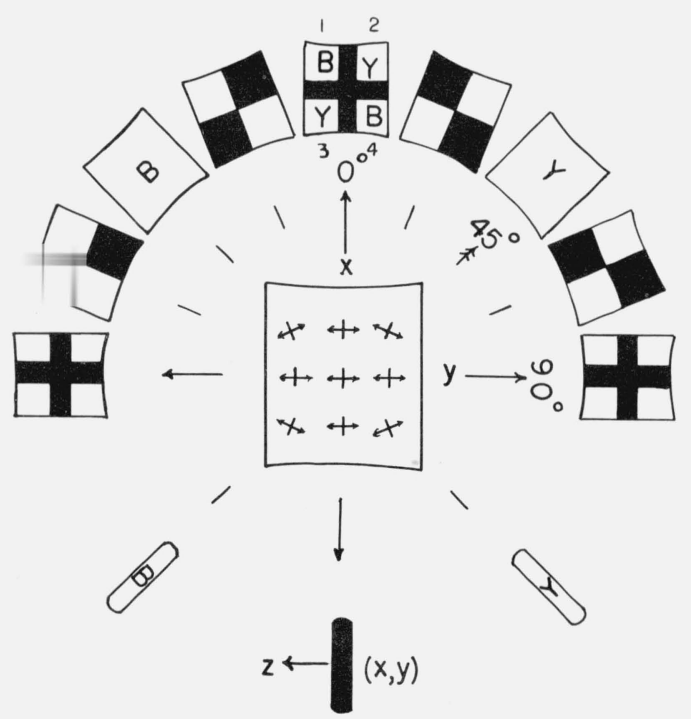

FIGURE 27. Schematic representation of birefringent properties of the larger dendrites shown in figures 15 to 21.

Shaded areas denote regions of extinction between crossed polarizers whose privileged directions of vibrations are horizontal and vertical in the diagram. $B=2$ nd order blue and $\mathrm{Y}=1 \mathrm{st}$ order yellow interference colors observed when a lst order red compensator is inserted between dendrite and analyser. Slow privileged direction of compensator is parallel to arrow pointing to $45^{\circ}$ position. The orientation of the slow direction of vibration of light traveling at right angles to the $x, y$ cross section of the dendrite is indicated by the arrowed arms of the crosses in the central diagram. ference colors observed after insertion of a first order red retardation plate between the crystals and the analyzer, are summarized in figure $27 .^{9}$ For the sake of brevity in the ensuing discussion, the long and short axes which bisect the plane of the dendrites will be referred to as the $x$ and $y$ axes respectively, as shown in figure 27 . The axis normal to the dendrite plane (i.e., the $x, y$ plane) is denoted $z$.

When viewed along axes parallel to the $x, y$ plane, the dendrites exhibit positive birefringence with respect to that plane (note interference colors when $x, y$ plane is parallel to $\pm 45^{\circ}$ positions in lower half of fig. 27) and become extinguished when the latter plane is oriented parallel to the privileged directions of the crossed polarizers $\left(0^{\circ}\right.$ and $90^{\circ}$ positions, lower part of fig. 27). When viewed along the $z$-axis, the dendrites exhibit throughout negative birefringence with respect to the $x$-direction (cf. interference colors with compensator inserted when $x$ is at $\pm 45^{\circ}$ to the privileged directions of the crossed polarizers, upper half of fig. 27). Consideration of the extinction characteristics and interference colors observed as a dendrite is rotated through $180^{\circ}$ about the $z$-axis indicates however that whereas the slow privileged direction of vibration of light which has traversed the crystal is oriented normal to the $x$-axis in the regions situated along and adjoining the $x$ and $y$ axes, it deviates symmetrically by up to $\pm 22 \frac{1}{2^{\circ}}$ from that orientation in the regions close to the apices. The sense of this deviation in orientation of the slow privileged direction of vibration near each of the four apices is as indicated in the central diagram in figure 27.

The extinction characteristics of the smaller dendrites (from the same preparation as those shown in figs. 22 to 26) were also examined under the polarizing microscope. So far as could be ascertained, considering their small size, their optical properties were essentially similar to those of the larger dendrites. The deviation in extinction angle between the axial regions and the apices appeared to be somewhat less pronounced (i.e., $<22^{1 / 2^{\circ}}$ ) than was the case in the larger dendrites.

Assuming that the refractive index ellipsoid of monoclinic polypropylene is uniaxial and positive and that the major axis of the ellipsoid is parallel (as indicated by calculations based on bond polarizabilities [18]) to the c-axis of the unit cell (i.e., parallel to the axes of the helical molecules), the positive birefringence exhibited by the dendrites with respect to the $x, y$ plane (lower part of fig. 27) indicates that the chain molecules are preferentially oriented parallel to that plane. This was substantiated by an electron diffraction pattern (fig. 28a) obtained from a dendrite oriented edge-on (fig. 28b) with respect to the electron beam. In view of the opacity of the interior of the dendrite to the

\footnotetext{
${ }^{9}$ The same general characteristics depicted in figure 27 were exhibited by (i) as-grown crystals in suspension in amyl acetate, (ii) crystals which had been dried and reimmersed in either amyl acetate or Cargille immersion liquids having refractive indices in the range $1.440-1.560$, (iii) as-grown crystals in suspension in amyl acetate diluted with an excess of
} Cargille immersion liquids having refraction indices in the range indicated in (ii). 


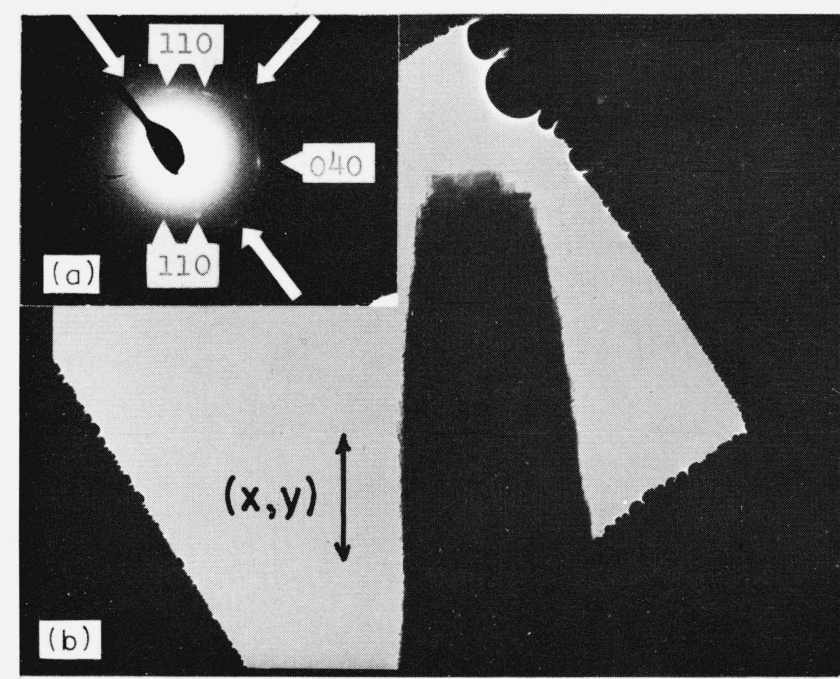

FIGURE 28. (a) Insert: Electron diffraction pattern from a dendrite oriented edge-on with respect to the electron beam. (b) Corresponding electron micrograph, $\times 7,267$, of portion of dendrite giving rise to diffraction pattern in $(a)$.

This dendrite was formed under the same conditions as those shown in figures 15 to 25 , the preparation was however filtered isothermally after being held for $18 \mathrm{hr}$ at $114^{\circ} \mathrm{C}$ Angle of rotation of image with respect to diffraction pattern is $8^{\circ}$ counterclockwise. Unmarked arrows indicate position of weak 130 spots. Disposition of diffraction spots indicates that the $\mathbf{b}$-crystallographic axis is oriented horizontally and the $\mathbf{c}$-axis at right angles to the field of view.

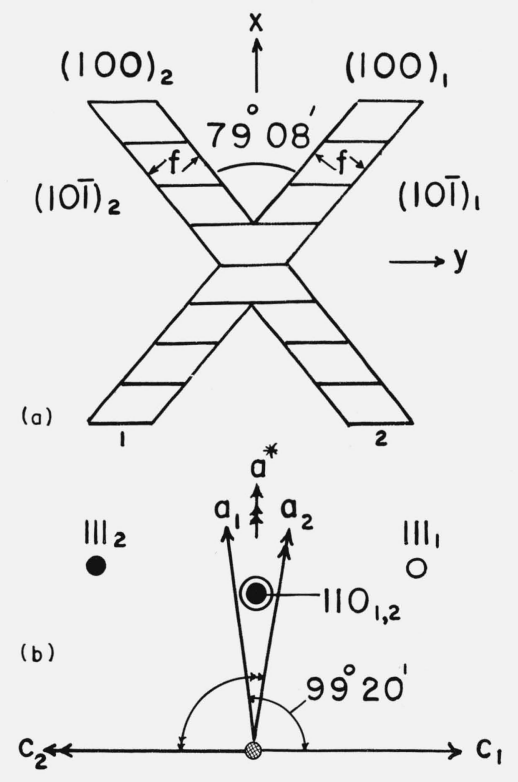

O

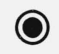

FIGURE 29. (a) b-axis projection of Model I twinned dendrite. Striations in branches indicate orientation of chain segments in each branch. (b). Diagram showing relative lattice orientations in twinned branches in (a), as well as projected disposition of 110 and 111 reciprocal lattice points $\left(\mathrm{d}_{110}=6.25 \AA, \mathrm{d}_{111}=4.2 \AA\right)$ with respect to the dendrite.

$\bigcirc$ Reciprocal lattice points corresponding to $\mathbf{a}_{1} \mathbf{c}_{1}$. Reciprocal lattice points corresponding to $\mathbf{a}_{2} \mathbf{c}_{2}$. impinging electrons, the pattern may be readily attributed to the transparent branches (note their lamellar character) protruding at its extremity. The diffraction spots recorded in figure 28a are reflections from $\{110\},(040)$, and $\{130\}$ planes. The disposition of the spots corresponds to that to be expected from a monoclinic crystal of polypropylene oriented with its c-axis parallel to the electron beam, i.e., parallel to the $x, y$ plane of the dendrite in figure $28 \mathrm{~b}$, and with its b-crystallographic axis (note the disposition of the 040 spot) essentially at right angles to both the beam direction and the $x, y$ plane.

On the basis of the latter observations, i.e., that the $\mathbf{b}$-axis in the dendrite branches is oriented at right angles to the $x, y$ plane of the dendrite, and that the c-axis in the branches is oriented parallel to that plane, it follows that the a-axis in individual branches must also lie parallel to that plane. In this respect, two crystallographically distinguishable but morphologically similar hypothetical "twinned" dendritic crystals may be envisaged whose respective morphologies would coincide closely with the experimental observations described above. The $\mathbf{b}$-axis projections of these two hypothetical models I and II, based on the unit cell parameters given by Natta et al., [3], are depicted schematically in figures $29 \mathrm{a}$ and $30 \mathrm{a}$. The corresponding relationships between the orientation of the a- and $\mathbf{c}$-axes in their constituent branches are
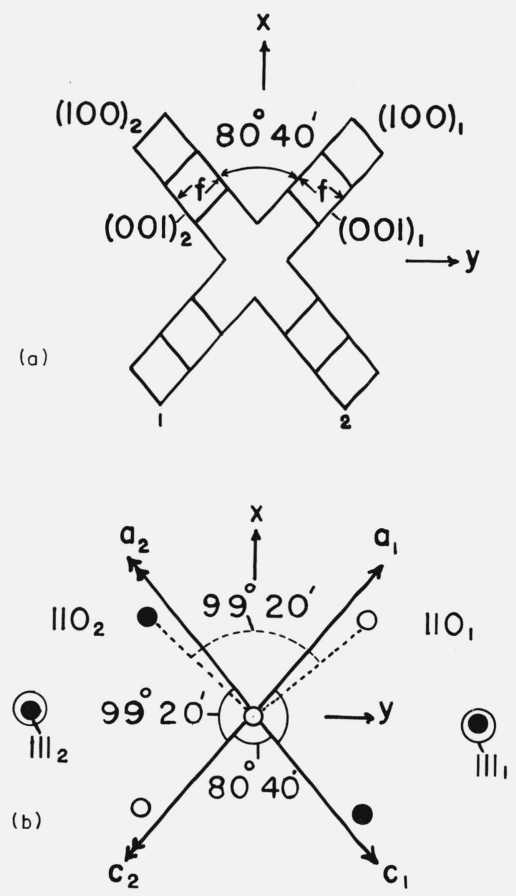

FIGURE 30. (a). b-axis projection of Model II twinned dendrite. (b) Diagram showing relative lattice orientations in twinned branches in (a) as well as projected disposition of 110 and 111 reciprocal lattice points with respect to the dendrite.

$\bigcirc$ Reciprocal lattice points corresponding to $\mathbf{a}_{1} \mathbf{c}_{1}$. - Reciprocal lattice points corresponding to $\mathbf{a}_{2} \mathbf{c}_{2}$. 
shown in figures $29 \mathrm{~b}$ and $30 \mathrm{~b}$. The fold surfaces (f) of individual branches are viewed edge-on in both models as depicted in figures 29a and 30a. In both models the acute dihedral angle between the fold surfaces of the intercrossing branches is circa $80^{\circ}$ (cf. experimentally estimated angle $\sim 85^{\circ}$ ). In order to compare the essential features of these models with respect to experimental observations we identify the axes bisecting the acute and obtuse angles between the dendrite branches as the $x$ and $y$ reference axes respectively (cf. the $x$ - and $y$-axes which have been referred to previously in the discussion of the experimentally observed optical properties of the dendrites (fig. 27)).

The essential and contrasting features of models I and II are summarized below:

Model I. The dominant feature of this model is that the axes of the constituent helical molecules, and hence the $\mathbf{c}$-axis, in all the branches are oriented throughout the whole structure parallel to $y$. The $\mathbf{a}^{*}$ and [001] axes in the "intercrossing" (twinned) branches in this model are parallel to $x$ and $y$ respectively. The respective lattice orientations in the twinned branches are such as to be brought into coincidence by rotation through $180^{\circ}$ either about $x$ (i.e., $\mathbf{a}^{*}$ ) as shown in figure 32 or about $y$ (i.e., [001]). It may be noted that $\mathbf{a}^{*}$ which is normal to the $\mathbf{b}$-axis in the monoclinic unit cell of polypropylene, is very closely parallel to the [601] axis in the cell, and that the (106) planes are very closely normal to the (100) planes. Individual branches in this model are bound by $(10 \overline{1}),(100)$ and $(010)$ faces (the latter which are parallel to the $x, y$ cross section are not shown in fig. 29a). The (101) faces, which are the fold surfaces, are denoted (f) in figure 29a.

Model II. In this model (fig. 30) it is assumed that the fold surfaces are parallel to the (001) planes in each branch whose other faces are parallel to the (100) and (010) planes. It is also assumed that the (100) planes in individual branches are parallel to the (001) planes in branches that "cross" them. The chain molecules in intercrossing branches are symmetrically inclinded at $49^{\circ} 40^{\prime}$ to $y$ in the $x, y$ plane, and the twinned lattice orientations are such as to be brought into coincidence by rotation through $180^{\circ}$ about $x$.

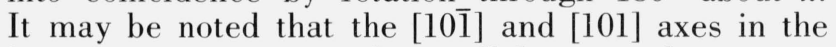
branches are very nearly parallel to $x$ and $y$ respectively.

Both Models I and II conform with the experimental observation that the $\mathbf{b}$-axis in the dendrites is oriented at right angles to their $x, y$ plane. That the overall development of the dendrites is slowest at right angles to the latter plane may be accounted for in both models if it is envisaged that the growth of individual branches occurs more rapidly along the direction normal to their (100) faces (i.e., along $\mathbf{a}^{*}$ ) than along [010].

In summary, therefore, on the basis of the two models discussed above, the possibility was envisaged, as an initial guide, that the mechanism of propagation of the dendritic monoclinic polypropylene crystals may involve either repeated twinning through $180^{\circ}$ lattice rotations about $\mathbf{a}^{*}$ or [001] as in figure $29 \mathrm{a}$, or about an axis at right angles to the b-crystallographic axis and almost coincident to $[10 \overline{1}]$ as in figure $30 \mathrm{a}$.

Using the two model twinned crystals I and II as a basis of reference, we turn now to a consideration of the experimentally observed optical properties of the dendrites about their $x, y$ cross section (upper half of fig. 27), in conjunction with some electron diffraction data obtained from their peripheral regions with the crystals oriented with their $x, y$ cross section at right angles to the electron beam.

The optical feature of the dendrites which initially strikes attention is the overall negative birefrigence they exhibit with respect to their $x$-axis since, a priori, this feature is consistent with the orientation of the chain molecules in Model I. The birefringence with respect to the $x$-axis was measured however, and found to be of the order of -0.007 birefringence units. ${ }^{10}$ This value is rather lower than would be expected when considered in the light of birefringence-draw ratio data obtained by Keedy et al., [19] from drawn filaments of isotactic polypropylene. A value closer to -0.03 birefringence units with respect to $x$ would be expected of a structure corresponding to Model I.

While the low birefringence of the crystals with respect to $x$ argues against Model I this feature alone could not be used to discriminate unambiguously against this model in view of the uncertainty involved in the determination of the overall thickness of the dendrites at right angles to the $x, y$ cross section, coupled with the fact that these structures are not solid objects and that consequently their effective thickness is most probably lower than the overall thickness which was used to calculate their birefringence.

A further factor adding to the uncertainty in interpreting the low value of the observed birefringence unambiguously in terms of a precursor model is the fact that the extinction behavior of the dendrites, which is not uniform throughout their $x, y$ cross section (fig. 27), indicates deviations (albeit symmetrical) in average molecular orientation about that cross section. We turn, therefore, to the outcome of electron diffraction experiments which, although subject to complications associated with the latter factor, nevertheless provide a more direct means of crystallographic characterization.

Two typically weak electron diffraction patterns obtained from smaller dendrites (same preparation as the crystals shown in figs. 22 to 25) are shown in figures 31 and 32 . Interpretation of these patterns is provided by consideration of figure 33 in which the $\mathbf{b}$-axis projection of the monoclinic unit cell of polypropylene is depicted together with the corresponding projection of the reciprocal lattice. The projected dispositions of the 110 and 111 reciprocal

${ }^{10}$ These measurements were made on the larger dendrites from the same preparation as those shown in figure 15. The dendrites in suspension in amyl acetate were deposited on a glass slide. Partial evaporation of excess solvent was allowed to occur in air after which an excess of a Cargille immersion liquid whose refractive index (1.510) corresponded to the isotropic refractive index of highly crystalline unoriented polypropylene [2] was added to the deposit. Possible contributions of form birefrigence were thus minimized. The birefrigence measurements were carried out with a Berek compensator. 

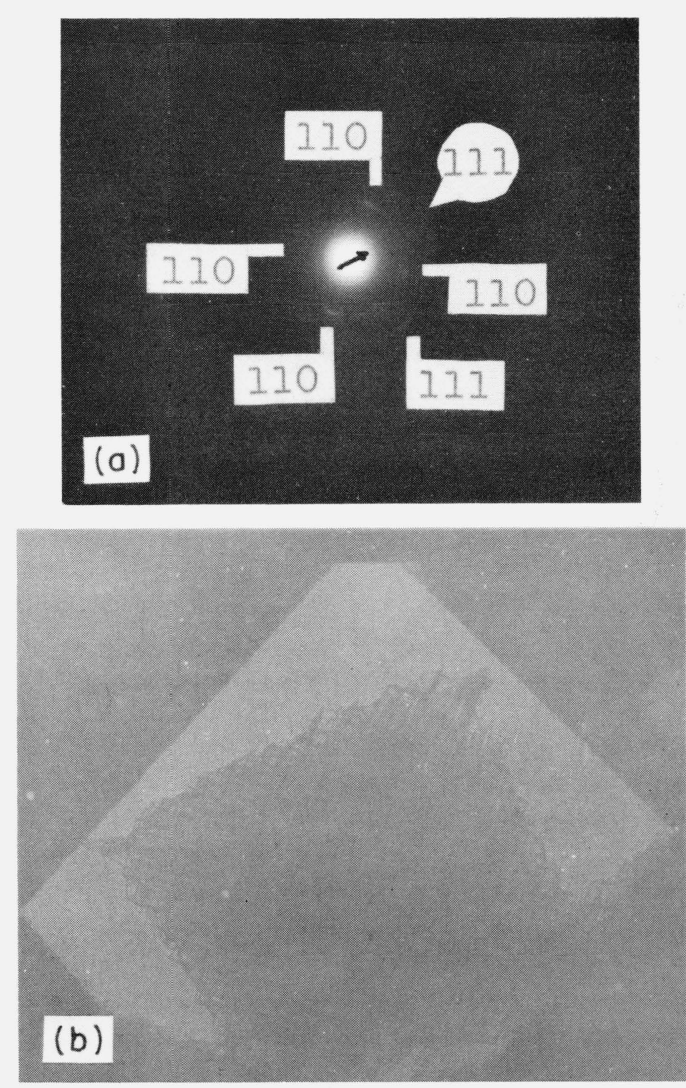

FIGURE 31. (a) Electron diffraction pattern obtained from the dendrite shown in $(b)$. Correction made for relative rotation of the image with respect to the diffraction pattern.

Central arrow in (a) indicates orientation of $x$-axis of dendrite with respect to the diffrac tion pattern. Magnification in (b) $\times 14800$.

lattice points with respect to the unit cell (real space) are also shown in the same figure. The angles of inclination of the (110) and (111) real space planes with respect to the ac plane of the unit cell are $73^{\circ}$ and $80^{\circ}$, respectively. In anticipation of what follows the projected dispositions of the 110 and 111 reciprocal lattice points with respect to the $\mathbf{b}$-axis projections of the model twinned crystals I and II shown in figures 29 and 30 may be considered.

The diffraction patterns shown in figures 31 and 32 have one particular feature in common, namely, in both cases four inner diametrically paired 110 reflections $(d=6.28 \AA)$ are discernible. The acute angle between the two pairs is $85^{\circ}$ in figure 30 and $\sim 62^{\circ}$ in figure 31 , some uncertainty being involved in the estimation of the latter angle in view of the arced and unevenly spotty nature of the reflections; hence the choice of the midpoints of the ares is subject to some arbitrariness. The feature of interest which will be returned to presently is the apparently twinned disposition of these 110 reflections, the bisector of the acute angle between the two pairs being closely coincident in orientation with the $x$-axis of the dendrites.

In addition to the 110 reflections, individual (i.e., unpaired) spots or arcs corresponding to an interplanar
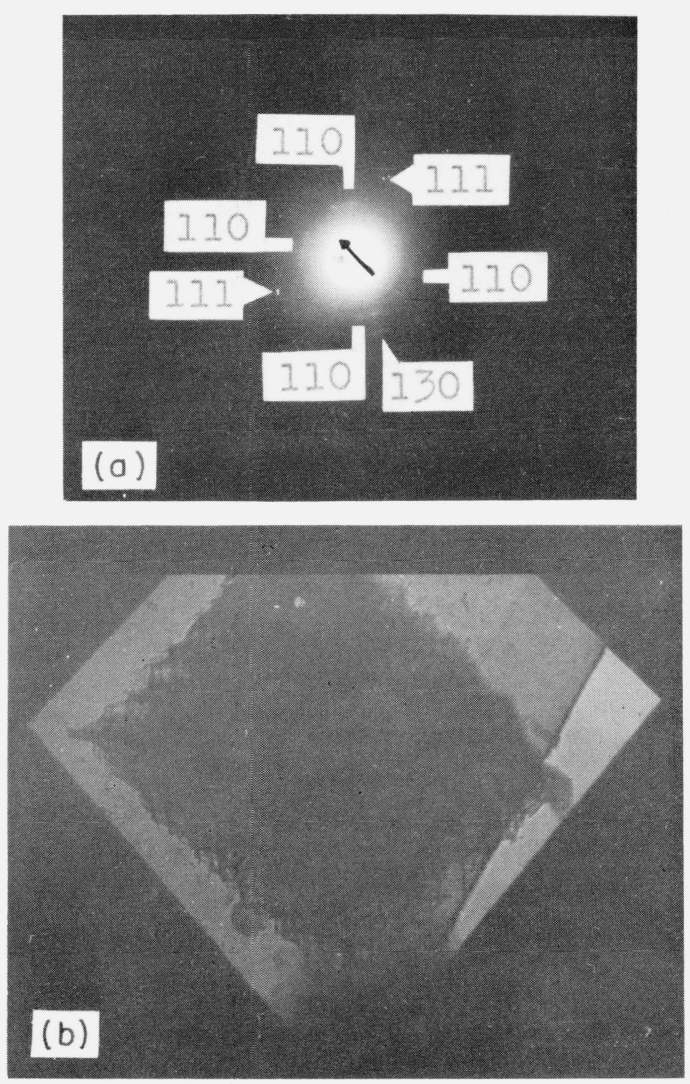

FIGURE 32. (a) Electron diffraction pattern obtained from dendrite shown in $(b)$. Correction made for relative rotation of the image with respect to the diffraction pattern.

Central arrow in (a) indicates orientation of $x$-axis of dendrite with respect to the diffraction pattern. Magnification in (b) $\times 14800$.

spacing of 4.15-4.20 $\AA$ which could thus be indexed 111 are pointed out in figures 30 and 31 . An individual arced reflection corresponding to a spacing of $4.75 \AA$, hence indexed 130 , may also be seen in figure 31 .

Some complicating factors arising from the very nature of the dendrites must be contended with in attempting to interpret the diffraction patterns in figures 31,32 , and the variations between them. First, the weakness of the patterns, which, as indicated earlier, could not be discerned visually on the fluorescent screen of the electron microscope, indicates that they are essentially contributed to only by the extremities of those branches that extend to the thinner peripheral regions of the dendrites. Second, it can be anticipated that distortions or relative displacements undergone by the branches in the peripheral regions as a consequence of surface tension forces incurred during drying could give rise to a situation approximating that of precessing the undistorted structure with respect to the electron beam. In principle, if the a- and c-axes in the branches are oriented parallel to the $x, y$ cross section, the (110), (111) and (130) planes would not satisfy the Bragg condition for diffraction (Bragg angle $<1^{\circ}$ for an $80 \mathrm{kv}$ electron 


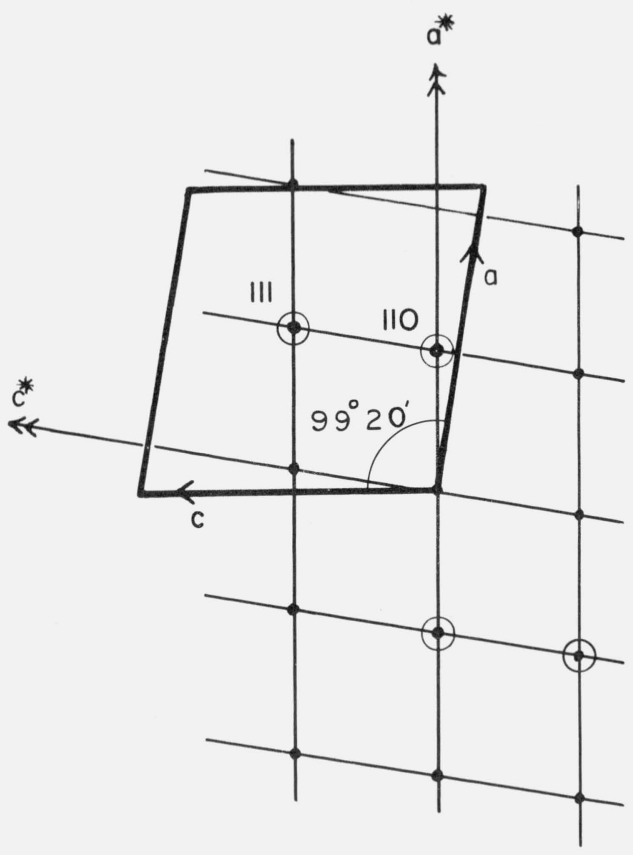

FIGURE 33. b-axis projection of monoclinic unit cell of poly. propylene, and corresponding projection of the reciprocal lattice indicating projected disposition of the 110 and 111 reciprocal lattice points.

$\mathbf{b}^{*}$ which is parallel to $\mathbf{b}$ is normal to the plane of the diagram. $\left(d_{110}=6.25 \AA, d_{11}\right.$ $=4.2 \AA$ ).

beam). The presence of these reflections can, however, be attributed to distortions in the network of branches; their resulting dispositions, however, would not be expected to coincide precisely with precession patterns (about the b-axis) corresponding to either models I or II. Some deductions may nevertheless be based on the disposition of the 110 arcs in figures 31 and 32 , with respect to the $x$-axis of the dendrites.

If the structure of the dendrites corresponded with Model I the 110 reflections from the twinned branches would coincide and be expected to fall along, or be spread about, an axis parallel to $x$ as may be deduced from figure 29b. The diffraction patterns shown in figures 31 and 32 in which the angle between the paired 110 reflections are $85^{\circ}$ and $\sim 62^{\circ}$ respectively therefore argue against Model I. The corresponding angle for Model II however is $\sim 100^{\circ}$. The discrepancy observed in the angle between the 110 reflections with respect to Model II may be attributed to distortions incurred by the crystal on drying, evidence for which may be detected in the electron micrographs shown in figures 31,32 , and, in a secondary way, to possible as-grown misorientations between the dendrite branches (see sec. 5 for discussion concerning possible origin of such misorientations). The same factors could also account for the fact that the reflections marked 111 on rectangular backgrounds in figures 31 and 32 do not fall along the axis parallel to $y$ as

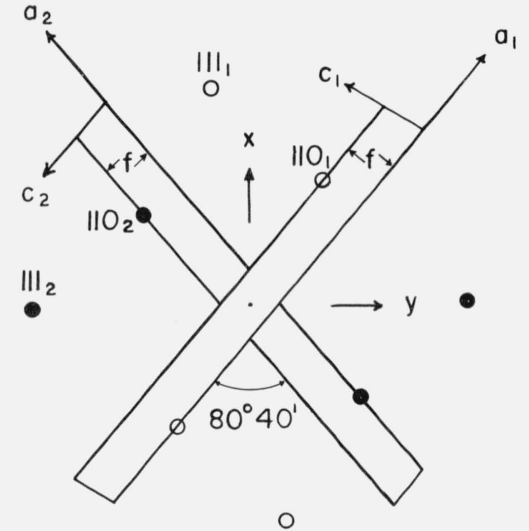

FiguRE 34. Schematic representation of the $\boldsymbol{b}$-axis projection of "twinned" branches in which the angle between their respective fold surfaces $(f)$ is $80^{\circ} 40^{\prime}$, their lattice orientations being related by an $80^{\circ} 40^{\prime}$ rotation about $\boldsymbol{b}$.

The projected disposition of the 110 and 111 reciprocal lattice points is also shown. Points corresponding to $\mathbf{a}_{1} \mathbf{c}_{1}$. Points corresponding to $\mathbf{a}_{2} \mathbf{c}_{2}$.

would be expected of Model II. In some instances however as indicated for example in figure 31, arced 111 reflections (circular background) included in the acute angle between the paired 110 reflections were observed. Unless it is assumed that they are artifacts associated with deformations in the dendrites, they raise the possibility that the lattice orientations in intercrossing branches may be related by an $80^{\circ} 40^{\prime}$ rotation about the $\mathbf{b}$-axis as shown in figure 34 . It should be noted in the latter instance, however, that whereas the chain axes in the twinned branches are respectively oriented at $80^{\circ} 40^{\prime}$ to one another as is the case in Model II, they are not symmetrically inclined with respect to $x$. Furthermore, whereas the chain molecules in branch (1), figure 34, are parallel to the fold surfaces of branch (2), the reverse does not hold, thus implying the unlikely possibility that there exist two different modes of formation of offspring branches from parent branches. Assuming, for reasons which will be discussed later (see sec. 5), that the formation of offspring branches from parent branches occurs through the epitaxial deposition of chain molecules on and parallel to the fold surfaces of parent branches, the development of branches (2) from branch (1) can be envisaged. The formation of branch (1) from branch (2) does not comply with the above assumption which is however satisfied by model II.

In summary, while realizing that the interpretation of the twinned nature of the dendrites presented in previous paragraphs is subject to ambiguities associated with the problem that they are not perfectly rigid entities, it appears nevertheless that the structure of these crystals corresponds closely to that depicted in Model II shown in figure 30. It is of interest to note in this respect that Geil [11] has reported that electron diffraction patterns obtained from those areas in very thin melt crystallized polypropylene 
films which exhibit a woven morphology indicate that the chain molecules lie parallel to the plane of the films and that the a-axis in the interweaving structural units is either parallel or perpendicular to the long axis of these units, the orientation being indeterminate in view of the interwoven disposition of the units. On the basis of the findings reported in the present paper it can thus be concluded that the a-axis in the individual interweaving units is parallel to their long axis and that the c-axis is transverse to their length. Reciprocally, Geil's observation lends further credence to Model II. In addition, following our observation that the polypropylene dendrites described herein could be grown from solutions of polypropylene in amyl acetate, Sauer, Richardson, and Morrow [20] have observed that similar dendrites exhibiting however a much more open structure (i.e., less profuse branching) could be grown from very dilute solutions of the polymer in $\alpha$-chloronaphthalene. These structures collapse and distort pronouncedly on drying with the result that individual lathlike lamellar branches protruding from the collapsed dendrite and lying with their fold surfaces parallel to the substrate were observed. Electron diffraction patterns from such lamellae as reported by Sauer et al., show that the chain molecules are oriented at right angles (or closely so) to the plane of the lamellae and that the a-axis is parallel to the long axis of the laths. These features are consistent with Model II.

\section{Discussion and Conclusions}

The coincidence between the overall shape of the dendritic polypropylene crystals described in section 4 with that of the earliest microscopically detectable apparently polyhedral objects which are observed to develop during the initial stages of growth of monoclinic spherulites (sec. 3.2b) leads to the conclusion that their fine structures must be similar. In short, it is deduced on the basis of the present study that monoclinic polypropylene spherulites evolve from initially formed dendritic crystal precursors whose fine structure and mode of propagation is similar to that of the highly unusual crystals shown in figures 15 to 26 .

The diagram shown in figure 35 represents schematically the respective profiles of the smaller and larger dendrites described in section 4. Also shown in the same figure is the corresponding cross-sectional profile exhibited by the transient and still essentially planar structures whose development corresponds, as shown in section 3 to a stage in the evolution of a monoclinic spherulite that is intermediate between the initially formed pseudopolyhedral precursor (dendritic crystal) and the ultimate spherulite.

Consideration of figure 35 clearly reveals the specific manner in which the dendritic precursor changes progressively in shape as it evolves into a spherulite. This feature suggests that the characteristically observed increase in concavity of the shorter "edges" and convexity of the longer edges of the dendritic

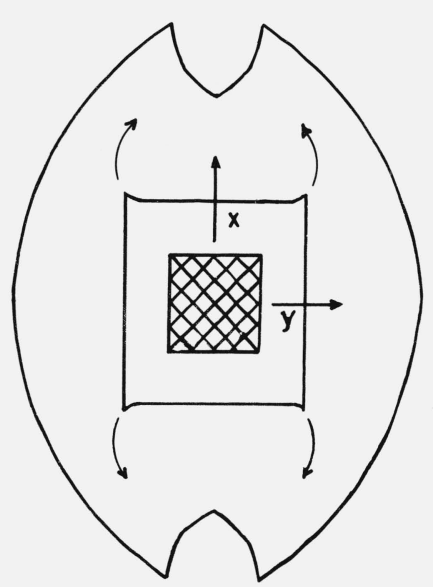

FIGURE 35. Comparison of $x, y$ cross sections of smaller and larger dendrites with that of the corresponding cross section of a notched aggregate representative of a more advanced stage in the evolution of a monoclinic spherulite.

precursor, followed by the characteristic symmetrical pincer pattern of filling in at the concave extremities leading to an intermediate elliptically profiled aggregate, must be the result of a progressive degeneration of the apparently regular pattern of propagation through branching associated with the precursor initially.

Before proceeding into a further discussion of the unusual habit of the dendritic precursors of the monoclinic spherulites, and the apparently specific manner in which they degenerate into spherulites, it is instructive first to consider the observations reported in previous sections in a more general perspective. With this purpose in mind, current views relating to the mechanism of formation of polymer spherulites are summarized below. This will serve further to emphasize the nature of the atypical features associated with the formation of monoclinic polypropylene spherulites.

The more common morphological forms adopted by crystalline polymers may be classified into essentially three categories. These are in ascending degree of morphological complexity: lamellar chain-folded single crystals (planar or hollow pyramidal), axialites, and spherulites.

The term axialite was first coined by Bassett, Keller, and Mitsuhashi [18] to a species of solution-grown crystalline aggregates of polyethylene which consist of chain folded lamellae splaying about a common axis parallel to the $\mathbf{b}$-crystallographic axis of the lamellae. When viewed along this common axis the axialites exhibit a sheaflike appearance, whereas they exhibit polygonal or oval shaped profiles when viewed at right angles to that axis. The contrasting appearance of these axialites when viewed in different perspectives led to the suggestion [18] that the sheaflike objects from which polymer spherulites grown in thin films from the melt have been frequently observed to evolve, 
as well as the hedrites [21] also observed in thin melt crystallized films of several polymers [21, 22, 23], are axialite type structures seen respectively in different perspectives. This generalization has been substantiated and elaborated on further by Keith [24] in a paper in which the relations among the different types of morphological forms commonly exhibited by polymers are discussed, in the context of spherulite formation, in terms of the role played by fractionation and impurity segregation in the crystallization of polymers $[25,26]$.

According to Keith [24] axialites and spherulites represent respectively successive stages in the evolution of an initially formed lamellar chain-folded crystal growing (e.g., from the melt) under the influence of impurity species which give rise to cellulation at the growth fronts of the crystal along which the chain molecules accrue in a regularly folded manner. Morphological evidence supporting this view has been presented in the case of isotactic polystyrene in particular. It appears, as suggested by Keith [24], that the single crystal $\rightarrow$ axialite $\rightarrow$ spherulite pattern of evolution associated with the formation of spherulites in the latter polymer is, with minor variations, generally characteristic of polymer spherulites which exhibit, when fully grown, conventional radiating lamellar fine structures such as found in polyethylene, polyoxymethylene, polyoxyethylene and poly(4-methylpentene-1) [7]. The same growth scheme applies most probably to spherulites of the hexagonal crystalline modification of polypropylene.

It is evident that the pattern of evolution of monoclinic polypropylene spherulites differs in a fundamental manner from the lamellar single crystal (planar or hollow pyramidal) $\rightarrow$ axialite $\rightarrow$ spherulite pattern of spherulite formation summarized above. The origin of this difference as well as the atypical fine structures exhibited by monoclinic polypropylene spherulites $[8,11]$ is clearly traceable to the unorthodox nature of the dendritic crystal precursors described herein, from which such spherulites evolve. The factors governing the unusual habit of these dendritic crystals of polypropylene are still obscure. Some considerations relating to their mode of initial propagation and progressive degeneration into spherulites may nevertheless be speculated on.

The puzzling feature about these polypropylene crystals is, as indicated by the diffraction data, that the orientations of the chain axes in the two families of "intercrossing" branches in these crystals are not coincident. This feature represents a departure from the generally encountered compliance of the habits and mechanisms of growth of polymer single as well as twinned crystals [27] with the inherently characteristic parallel mode of chain packing which long chain compounds adopt in the crystalline state.

Clearly the $80^{\circ}$ change in chain orientation between parent and offspring branches implicit in twinned Model II (fig. 30) raises the evident question, namely, how does an offspring branch in which the chain segments (and fold surfaces) are oriented at $80^{\circ}$ to those in a parent branch develop from the latter in such a specific manner? One would expect that such a change in lattice orientation between parent and offspring branches would involve an interim boundary region of considerable disorder between them if one assumes that there exist a substantial number of tie molecules between individual branches and their offshoots. It is difficult to envisage that crystallographic information of a specific nature could be transmitted through such a region, particularly so in view of the $80^{\circ}$ change in chain orientation involved. In short, the mechanism of branching of the dendrites still remains obscure. It may be speculated, however, that the formation of new branches could be initiated by the epitaxial accretion of individual molecules in a regularly folded conformation onto the fold surfaces (i.e., the (001) faces) of parent branches such that the chain segments lie parallel to the latter faces. The resulting monomolecular "steps" formed by such individual molecules might then be envisaged to develop further through the parallel accretion of fresh molecules onto them. Such an epitaxial mode of development of new branches evidently presupposes that some topographical features of the (001) fold surfaces in the parent branches must favor the initial deposition of individual molecules parallel to these faces in a specific manner such that their chain segments are also oriented at right angles to the $\mathbf{b}$ crystallographic axis in the parent branch. This latter feature must be assumed in order to account for the parallel orientation of the $\mathbf{b}$-axis in both parent and offspring branches.

We turn now to a consideration of the nature of possible factors which lead to the progressive evolution of the dendritic crystal precursors discussed above into monoclinic spherulitic aggregates.

It has been pointed out earlier that the progressive transition in shape (see growth sequences in figs. 13, 14, and diagrammatic representation, fig. 35) which the dendrites undergo as they evolve into spherulites must be a consequence of progressive deviations from the apparent crystallographically specific pattern of branch propagation which characterizes their earliest stages of development.

Evidence has been obtained which indicates that the propagation of new lamellar branches from the fold surfaces of chain-folded parent lamellae in the manner depicted schematically in figure 26 remains however basically an intrinsic and continuing feature of the mechanism of evolution of the dendrites into spherulites. Briefly, it is pointed out in this latter respect that exploratory experiments have revealed that polypropylene crystallizes uniformly in the form of "notched" type aggregates, examples of which are shown in figure 36 , from 0.01 percent amyl acetate solutions at $100{ }^{\circ} \mathrm{C}$. Surface replicas ${ }^{11}$ of these aggregates of which the shape is similar to that of the transient structures which develop during the still

\footnotetext{
1 The preparation was filtered isothermally in the manner described earlier. The replicas were prepared by the same technique as that used for the dendrites described in section 4 .
} 


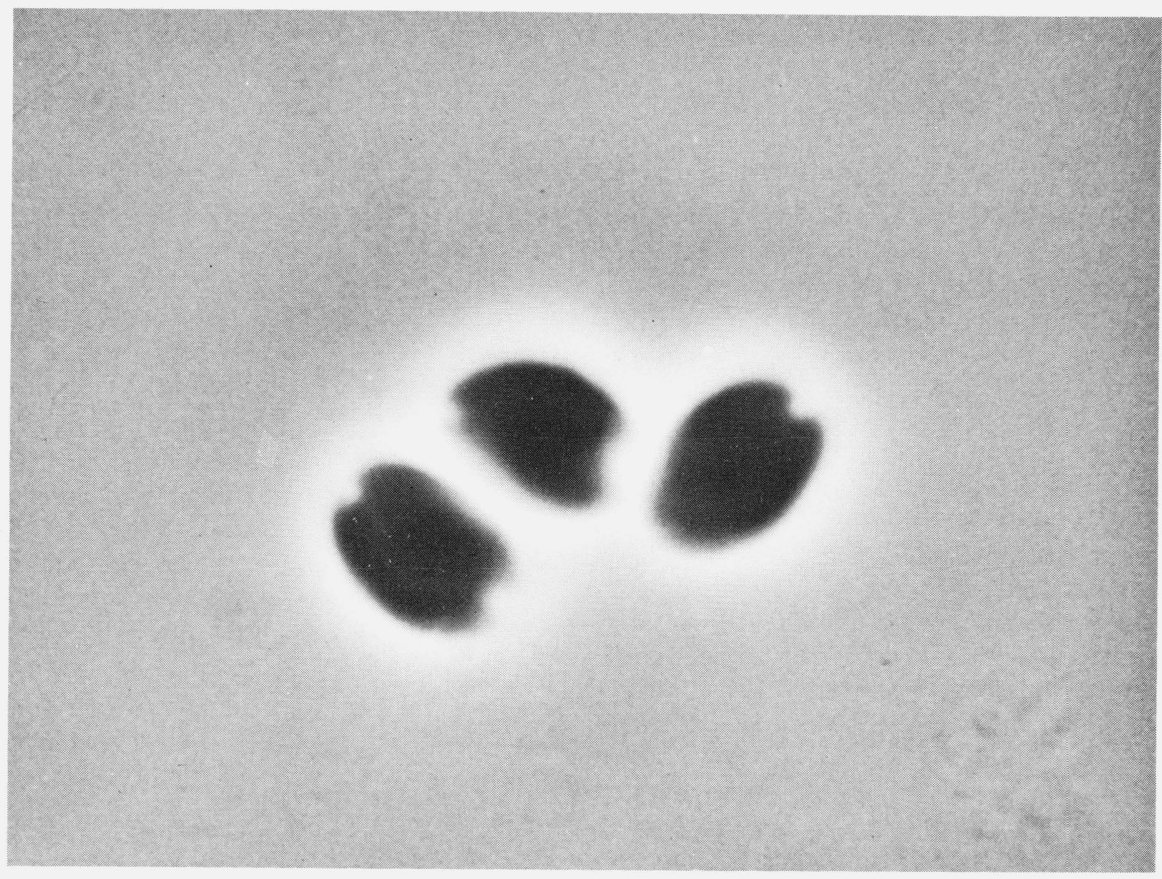

FIGURE 36. Notched aggregates grown from a 0.01 percent solution of polypropylene in amyl acetate at $100^{\circ} \mathrm{C}$ Phase Contrast $\times 2100$.

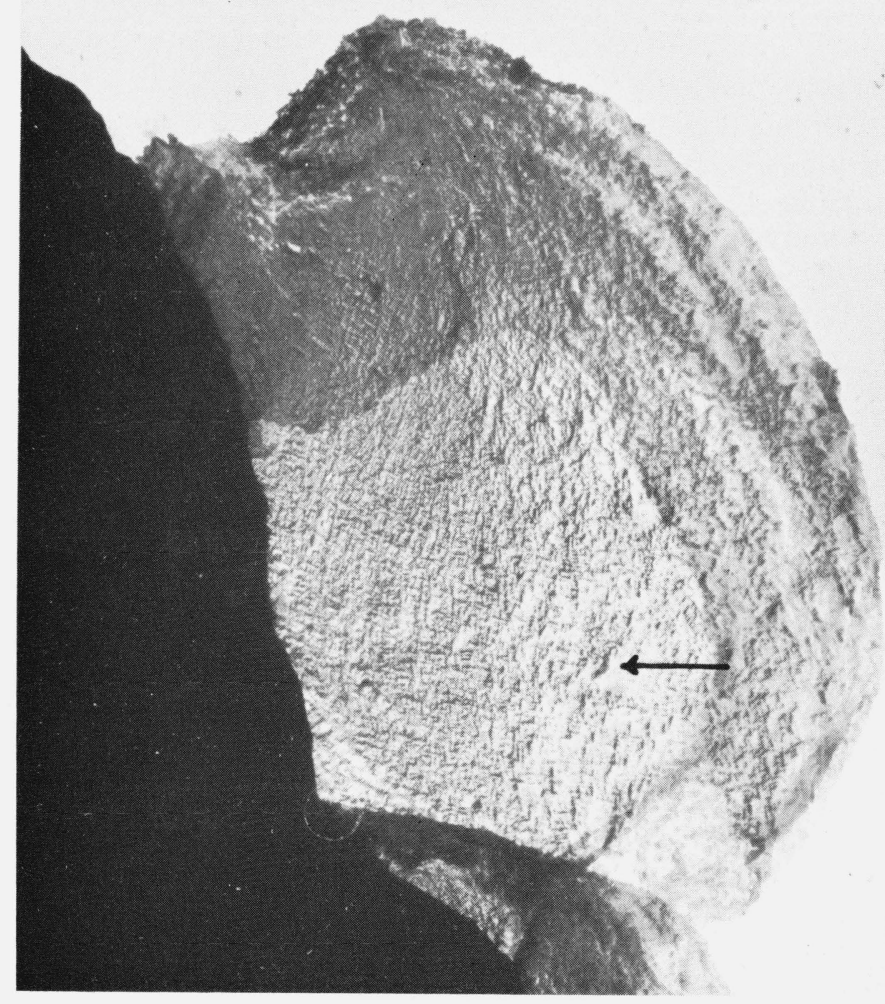

FIGURE 37. Replica of an aggregate similar to those shown in figure 36.

A cross-hatched fine structure is faintly discernible at this relatively low magnification. A cross-hatched fine structure is faintly discernible at this relatively low magnification.
Arrow points to mark identifying region shown at higher magnification in figure 38 . $\times 10800$. 


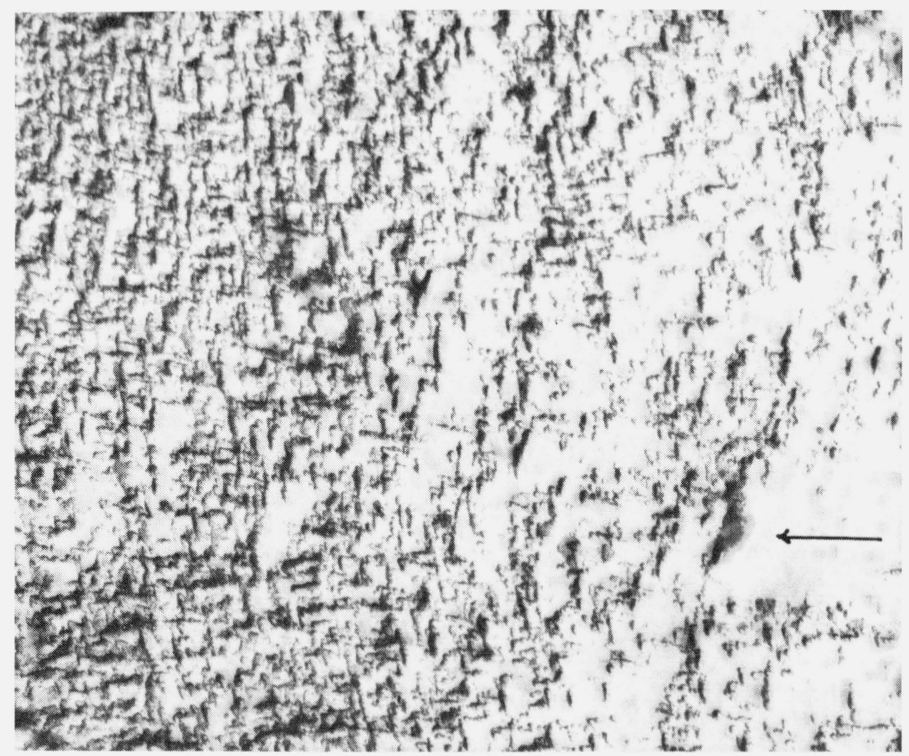

FigURE 38. A view at higher magnification of a portion (note arrow) of the replica shown in figure 37, revealing the cross-hatched surface texture of the replicated aggregate.

relatively early stages of evolution of the initial dendritic crystal precursors into spherulites revealed, as shown in figures 37 and 38 , a distinctly cross-hatched fine structure clearly related to that of the dendrites described in section 4 .

Two factors, one possibly intrinsically associated with the folded conformation of the chain molecules in the lamellar branches, and the other environmental, may be considered, that contribute to the progressive degeneration of the dendritic crystal precursors into spherulites.

With respect to the first of the two factors indicated above, recent quantitative measurements of lattice distortions arising from the chain folds in polymer crystals reported by Bassett [28] may be pointed out. These measurements have been derived from an analysis of Moiré patterns exhibited under the electron microscope by bilayer crystals of polyoxymethylene and poly(4-methylpentene-1) in particular. It has been shown that the spacing of a family of planes parallel to the chain molecules in crystals of these two polymers differs depending on whether or not the planes contain the chain folds. Such lattice distortions are attributed to the bulkiness of the folds which give rise to a slight increase in spacing between planes along which the molecules accrue in a regularly folded conformation. Bassett et al., [29] present evidence suggesting that the resulting distortion of the tetragonal subcell in simple (four-sectored) lamellar crystals of poly(4-methylpentene-1) causes the lamellae to adopt a very slightly curved ("conicalness"), as distinct from planar overall shape. It would appear that the highly curved bowl-shaped tetragonal crystals of poly(4-methylpentene-1) consisting of multisectored chain folded layers observed recently by Khoury and Barnes [30], as well as the orthorhombic crystals of polybutene-1 which, as reported by Holland and Miller [31], are curled up in the form of tubelike structures, owe their distinctly curved habits to lattice distortions associated with the chain folds in their constituent lamellae. It also seems very likely that the well established twisting of the constituent radiating lamellae in certain species of polymer spherulites $[32,33,34]$ is also a consequence of lattice distortions associated with chain folds [35]. In short, there exists a widespread body of evidence of the formation of chain-folded lamellae that are curved in some manner, as well as some direct and circumstantial evidence indicating that this phenomenon may be associated with lattice distortions due to the bulkiness of the folds in their constituent molecules.

On the basis of the phenomena indicated in the foregoing paragraph the possibility may be envisaged that the lamellar branches in the polypropylene dendrites may develop as very slightly curved rather than planar laths. Such an effect would clearly lead cumulatively to progressively more pronounced misorientations between newly formed branches throughout the dendrite and hence to a slow change in overall shape of the developing structure.

Direct detection of a possible very slight curvature of individual branches in the dendrites described in section 4 is clearly hindered by the impossibility of tracing individual branches unequivocally for appreciable distances. Deviations in the relative orientations of the dendrite branches may however be seen in the 
replicas of the larger dendrites shown in figures 20 and 21. In addition, the slight change in profile of the dendrites between the stages of growth corresponding to the smaller and larger crystals described in section 4, as evidenced by the more pronounced concavity of the shorter edges of the latter, coupled with the symmetrical deviations in extinction (about the $x, y$ cross section) between crossed polarizers, distinctly exhibited by the larger dendrites, suggests the contribution of a factor inherent in the very nature of the structure and mode of propagation of the dendrites to their progressive change in shape. It is merely pointed out in this respect, that a tendency of the four primary branches (i.e., those lying along the diagonals of the rectangular $x, y$ cross section of the dendrites) which play a major role in the propagation of the crystals, to curve inwards towards the interior of the crystal in the manner indicated in exaggerated fashion by the arrows in figure 35 would account for the characteristic manner in which the overall shape of the dendrites changes progressively during their early stages of evolution into spherulites.

We turn now to a brief discussion of the role played by environmental factors on the mode of propagation of the dendritic crystal precursors and their progressive evolution into spherulites under isothermal conditions. The influence of temperature, and hence rate of growth, as well as rejected impurity species (e.g., atactic molecules and low molecular weight chains) on the mode of propagation of the dendritic crystal precursors needs to be considered in this respect. Some indication of the effect of these factors may be obtained from a consideration of their influence on the development of individual dendrite branches. We may borrow in this respect from the views advanced by Keith [24] relating to the influence of impurity segregation at the growth fronts of a chain-folded polymer crystal.

It may be anticipated that whether growth occurs from solution or from the melt, an inpurity-rich layer surrounds the dendrite, thus leading to cellulation in the form of pronounced reentrances developing at those faces of each branch at which growth occurs through the parallel accretion of chain molecules (i.e., the (100) and (010) faces). Lattice misorientations between neighboring "cells" may then be expected to lead to the progressive acquisition of a polycrystalline character by each branch, as has been observed for example in the case of individual polystyrene chainfolded lamellae growing from the melt. Intrabranch lattice misorientations would evidently lead to misorientations between offspring branches growing from different regions of the fold surfaces of a parent branch and hence would contribute cumulatively to the progressive degeneration of the initial dendritic crystal into a spherulite.

Recapitulating, therefore, it is considered that the characteristic development of monoclinic spherulites from the particular species of dendritic crystals described in this paper is brought about cumulatively as a consequence of (a) the curving of individual dendrite branches due to lattice distortions associated with chain folds, and (b) the progressive acquisition of a polycrystalline character by individual branches growing under the influence of rejected impurity species. At low undercoolings and hence slow growth rates which favor the diffusion of impurities away from the growth fronts of the constituent dendrite branches the disruptive effects of cellulation are minimized in particular. Under such conditions the progressive transition from dendritic crystal to spherulite will be gradual and the initial precursor dendrite may attain, as has been observed, microscopic (optical) proportions before exhibiting pronounced signs of degeneration. At high undercoolings, however, the dendrite to spherulite transition would occur much earlier in the growth sequence at the submicroscopic level of resolution with the result that no initial pseudopolyhedral precursor will be observed.

The considerations presented in the preceeding three paragraphs have been only concerned with the influence of rejected impurity species on the degeneration of the dendritic precursors. An important consideration arises however regarding the influence of impurity rejected species on the frequency with which offspring branches develop from a parent branch in these dendritic precursors. In this respect the possibility cannot be discounted that the development of discrete offspring branches from the fold surfaces [(001) faces] of a parent lamella may actually result from the favored epitaxial accretion of molecules (assuming the epitaxial mode of development of branches suggested earlier holds) only on specific asperities on the fold surfaces of parent lamellae, and not necessarily due to a cellular pattern of monomolecular deposition of chains parallel to fold surfaces having an initial uniform topography. Carrying this latter conjecture further, it would follow that the frequency of branching will be governed by the number of branch-initiating asperities per unit area of the (001) faces, such as for example localized steps formed on those faces due to irregularities or fluctuations in the fold period of the constituent chain molecules in the parent lamella, which in turn may well be governed by the environmental conditions.

In conclusion, while the present study provides some detailed insight into the nature of the early stages of evolution of monoclinic polypropylene spherulites from solution as well as from the melt, a final analysis will have to account for the preferred molecular orientation with respect to the radial direction in the fully grown spherulites, information on which is, as indicated in the introduction, at present limited to monoclinic spherulites grown from the melt in the range of 110 to $148{ }^{\circ} \mathrm{C}[2]$. While such a detailed analysis is beyond the scope of the present study, it may be pointed out at this stage that the preferred tangential orientation of the b-crystallographic axis in melt grown spherulites [2] correlates with the observation that growth is slowest parallel to the b-crystallographic axis in the branches of the dendritic precursor crystals. The average $65^{\circ}$ to $70^{\circ}$ inclination of the chain molecules with respect to the radial direction in these spherulites [2] still remains unexplained at this stage. 
The author thanks E. Passaglia for the benefit of many helpful and critical discussions. Thanks are due to P. H. Geil for the loan of unpublished electron diffraction patterns.

\section{References}

[1] F. J. Padden, Jr., and H. D. Keith, J. Appl. Phys. 30, 1479 (1959).

[2] H. D. Keith, F. J. Padden, Jr., N. M. Walter, and H. W. Wyckoff, J. Appl. Phys. 30, 1485 (1959).

[3] G. Natta, P. Corradini, M. Cesari, Atti, accad. nazl. Lincei. Rend. Classe sci. fis. mat. e nat. 21, 365 (1956).

[4] E. J. Addink and J. Beintema, Polymer (London) 2, 185 (1961).

[5] A. Turner-Jones, Jean M. Aizlewood, and D. R. Beckett, Makromolekulare Chem. 75, 134 (1964).

[6] P. H. Geil, Polymer Single Crystals, ch. IV, p. 269 (Interscience, New York, 1963).

[7] P. H. Geil, Polymer Single Crystals, ch. IV, p. 223 (Interscience, New York, 1963)

[8] G. W. Bailey, J. Polymer Sci. 62, 241 (1962).

[9] A. Keller, Kolloid-Z. 165, 15 (1959).

[10] P. H. Geil, Polymer Single Crystals, ch. IV, p. 270 (Interscience, New York, 1963).

[11] P. H. Geil, Polymer Single Crystals, ch. III, p. 214-220 (Inter science, New York, 1963).

[12] A. Hiltz. Private communication.

[13] H. J. Leugering, Makromolekulare Chem. 68, 223 (1963).

[14] P. H. Geil, Polymer Single Crystals (Interscience New York, 1963, ch. III, p. 211).

[15] F. Khoury, Bull. Am. Phys. Soc. Series 11, 9, 275 (1964), Paper JA 4.

[16] D. C. Bassett and A. Keller, Phil. Mag. 7, 1553 (1962).
[17] R. Salovey and F. R. Dammont, J. Polymer Sci. Al, 2155 (1963).

[18] D. C. Bassett, A. Keller and S. Mitsuhashi, J. Polymer Sci. A 1, 763 (1963).

[19] D. A. Keedy, J. Powers and R. S. Stein, J. Appl. Phys. 31, $1911(1960)$

[20] J. A. Sauer, G. C. Richardson and D. R. Morrow, Bull. Am. Phys. Soc. Series 11, 10, 354 (1965) Paper EG8.

[21] P. H. Geil in Growth and Perfection of Crystals, p. 579 (John Wiley \& Sons, Inc., New York, N.Y., 1958).

[22] H. J. Leugering, Kolloid-Z 172, 184 (1960).

[23] F. Danusso and F. Sabbioni. Rend. Inst. Lombardo Sci. Lettere A92 435 (1958).

[24] H. D. Keith, J. Polymer Sci. 2A, 4339 (1964).

[25] H. D. Keith and F. J. Padden, Jr., J. Appl. Phys. 34, 2409 (1963).

[26] H. D. Keith and F. J. Padden, Jr., J. Appl. Phys. 35, 1270. 1286 (1964).

[27] P. H. Geil, Polymer Single Crystals, ch. II (Interscience New York, 1963).

[28] D. C. Bassett, Phil. Mag. 10, 595 (1964).

[29] D. C. Bassett, F. R. Dammont and R. Salovey, Polymer (London) 5, 579 (1964)

[30] F. Khoury and J. D. Barnes, Bull. Am. Phys. Soc. Series 11 10, 366 (1965) Papers HG3, HG4.

[31] V. F. Holland and R. L. Miller, J. Appl. Phys. 35, 3241 (1964).

[32] H. D. Keith and F. J. Padden, Jr., J. Polymer Sci. 39, 101, 123 (1959).

[33] F. P. Price, J. Polymer Sci. 39, 139 (1959).

[34] A. Keller, J. Polymer Sci. 39, 151 (1959).

[35] J. D. Hoffman and J. L. Lauritzen, Jr., J. Res. NBS 65A (Phys. and Chem.) No. 4, 297 (1961).

(Paper 70Al-385) 